Article

\title{
Towards the de novo Design of HIV-1 Protease Inhibitors based on Natural Products
}

\author{
Ana L. Chávez-Hernández ${ }^{1}$, K. Eurídice Juaréz-Mercado ${ }^{1}$, Fernanda I. Saldívar-González ${ }^{1}$ and José L. Medina \\ Franco ${ }^{1, *}$
}

1 DIFACQUIM Research Group, Department of Pharmacy, School of Chemistry, Universidad Nacional Autónoma de México, Avenida Universidad 3000, México City 04510, México; anachavez3026@gmail.com (A.L.C.-H.); kaeuridice@gmail.com (K.E.J.-M.); fer.saldivarg@gmail.com (F. I. S.-G.); medinaj1@unam.mx (J. L. M.-F.).

* Correspondence: medinajl@unam.mx; Tel..+52-55-5622-3899

\begin{abstract}
The acquired immunodeficiency syndrome (AIDS) caused by the human immunodeficiency virus (HIV) continues to be a public health problem. In 2020, 680,000 people died from HIV-related causes, and 1.5 million people were infected. Antiretrovirals are only a way to control HIV infection but not to cure AIDS. As such, effective treatment must be developed to control AIDS. Developing a drug is not an easy task, and there is an enormous amount of work and economic resources invested. For this reason, it is highly convenient to employ computer-aided drug design methods, which can help generate and identify novel molecules. Using the de novo design, new novel molecules can be developed using fragments as building blocks. In this work, we develop a virtual focused compound library of HIV-1 viral protease inhibitors from natural product fragments. Natural products are characterized by a large diversity of functional groups, many sp ${ }^{3}$ atoms, and chiral centers. Pseudo-natural products are a combination of natural products fragments that keep the desired structural characteristics from different natural products. An interactive version of chemical space visualization of virtual compounds focused on HIV-1 viral protease inhibitors from natural product fragments is freely available at https://figshare.com/s/ceb58d58e8f5585ce67e.
\end{abstract}

Keywords: artificial intelligence; de novo design; fragment-based drug discovery; HIV-1 inhibitors, pseudo natural products.

\section{Introduction}

The acquired immunodeficiency syndrome (AIDS) caused by the human immunodeficiency virus (HIV) is a major global public health concern. In 2020, the World Health Organization (WHO) reported that approximately 37.7 million people live with HIV out of 24.5 million from the African region. In 2020, 680,000 people died from HIV-related causes and 1.5 million people acquired it [1]. There is no definite treatment for AIDS. Therefore, it is necessary to collaborate to develop a treatment since the antiretroviral drugs currently approved by Food and Drug Administration (FDA) to clinical use only control AIDS and prevent HIV-1 transmission between individuals (Figure 1 and Table 1) [2-4].

Drug design and development demand many years of hard work and economic investment. Most drug candidates are prone to fail [5]. From 25,000 compounds that start in the laboratory, only 25 make it through preclinical testing to human testing, and just five of those reach the actual clinical use [6]. Computer-aided drug design (CADD) has contributed to yielding several drugs into the clinic, yet it has several challenges ahead [7]. Among the CADD methods, de novo design has gained relevance due to the diversity of structures generated by optimizing the algorithms used. From a methodological 
point of view, artificial intelligence as boosted the development and application of de novo design $[5,8,9]$.<smiles>CC(C)CN(CC(O)C(Cc1ccccc1)NC(=O)OC1CCOC1)S(=O)(=O)c1ccc(N)cc1</smiles>

Amprenavir $\mathrm{EC}_{50}=12-80 \mathrm{nM}$<smiles>CC(C)CN(CC(NC(=O)OC1CCOC1)C(O)(O)O)S(=O)(=O)c1ccc(N)cc1</smiles>

Fosamprenavir $\mathrm{EC}_{50}=12-80 \mathrm{nM}$<smiles>Cc1c(O)cccc1C(=O)NC(CSc1ccccc1)C(O)CN1CC2CCCCC2CC1C(=O)NC(C)(C)C</smiles>

Nelfinavir $\mathrm{EC}_{50}=30-60 \mathrm{nM}$

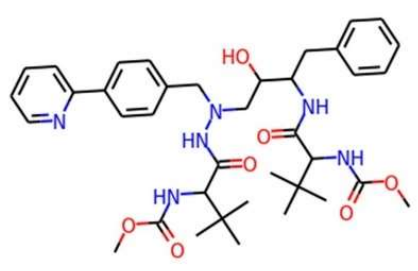

Atazanavir

$\mathrm{EC}_{50}=2.6-5.3 \mathrm{nM}$

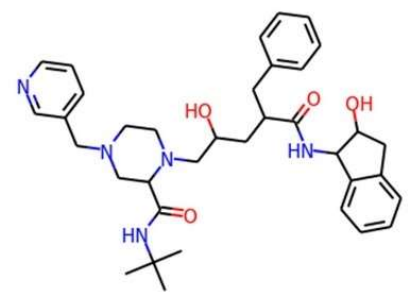

Indinavir

$\mathrm{EC}_{50}=5.5 \mathrm{nM}$<smiles>CC(C)c1nc(CN(C)C(=O)NC(C(=O)NC(Cc2ccccc2)CC(O)C(Cc2ccccc2)NC(=O)OCc2cncs2)C(C)C)cs1</smiles>

Ritonavir

$\mathrm{EC}_{50}=25 \mathrm{nM}$

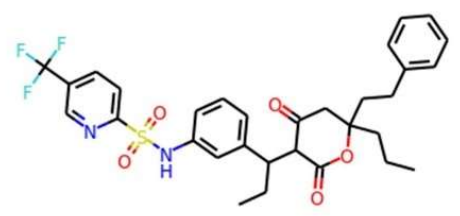

Tipranavir

$\mathrm{EC}_{50}=30-70 \mathrm{nM}$

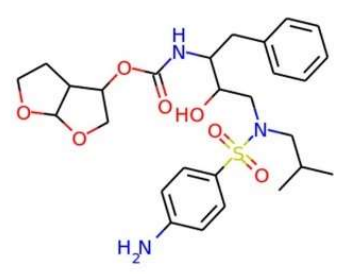

Darunavir
$E C_{50}=1-2 n M$

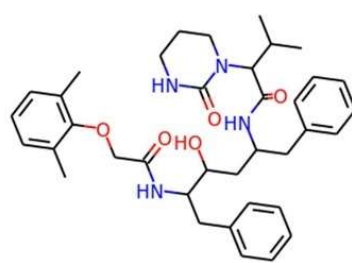

Lopinavir $\mathrm{EC}_{50}=17 \mathrm{nM}$

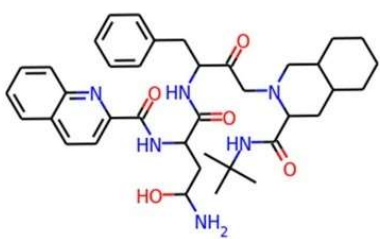

Saquinavir

$\mathrm{EC}_{50}=37.7 \mathrm{nM}$

Figure 1: Chemical structures of ten FDA-Approved HIV-1 protease inhibitors (Amprenavir, Atazanavir, Darunavir, Fosamprenavir, Indinavir, Lopinavir, Nelfinavir, Ritonavir, Saquinavir, Tipranavir). The EC50 is the concentration of drug required to produce $50 \%$ of the maximum possible effect. 
Table 1: FDA-Approved HIV-1 protease inhibitors which will be used as a reference for the de novo design of the new chemical compounds. ${ }^{a}$ Fosamprenavir is the phosphate ester prodrug of amprenavir.

\begin{tabular}{cccc}
\hline Generic name & Brand name & EC $_{50}[3]$ & FDA approval \\
\hline Amprenavir & Agenerase & $12-80 \mathrm{nM}$ & 1999 \\
Atazanavir & Reyataz & $2.6-5.3 \mathrm{nM}$ & 2003 \\
Darunavir & Prezista & $1-2 \mathrm{nM}$ & 2006 \\
Fosamprenavira & Lexiva & $12-80 \mathrm{nM}$ & 2003 \\
Indinavir & Crixivan & $5.5 \mathrm{nM}$ & 1996 \\
Lopinavir & Kaletra & $17 \mathrm{nM}$ & 2000 \\
Nelfinavir & Viracept & $30-60 \mathrm{nM}$ & 1997 \\
Ritonavir & Norvir & $25 \mathrm{nM}$ & 1996 \\
Saquinavir & Invirase & $37.7 \mathrm{nM}$ & 1995 \\
Tipranavir & Aptivus & $30-70 \mathrm{nM}$ & 2005 \\
\hline
\end{tabular}

The main goal of de novo design is to suggest novel molecular structures from scratch with desired activity on a pharmacological target and desired properties [10]. The new structures can be made using two general approaches: fragment-based and atom-based. The advantage of the fragment-based approach is that it narrows down the search in chemical space and maintains good chemical structure diversity [11-13]. Additionally, fragments form fewer interactions that should be able to bind to a greater number of sites on a greater number of proteins. Fragments are small (less than 20 heavy atoms) and typically soluble; they are likely to have better pharmaceutical properties as well as the new chemical compounds generated from them [14]. Over the last 20 years, four drugs from fragment-based drug discovery (FBDD) have been approved, and 40 compounds are currently in clinical trials [15].

Recently, de novo design and artificial intelligence have been combined to propose novel molecules for the treatment of SARS-CoV-2 based on HIV-1 protease and the approved drugs that inhibit this viral protease [8]. Another successful example of de novo design focusing on HIV research led to four molecules from a new compound library generated from the ZINC database [16]. Other approaches de novo design was based on enumerating libraries using chemical reactions $[17,18]$ and are also promising to expand the epigenetic relevant chemical space [19].

The development of new chemical compounds using de novo design can begin from natural product-derived fragments. Natural products have been attractive chemical compounds because they are characterized by a larger number of $\mathrm{sp}^{3}$ carbon atoms, chiral centers (associated with structural complexity), the larger scaffold diversity, and functional groups, hence their relevance for use as building-blocks [20,21]. In previous studies, we showed that natural products cover regions of chemical space that have not yet been explored by synthetically accessible compounds and those with biological activity [22]. For this reason, natural products could be used as building-blocks to develop novel synthetic molecules or pseudo-natural products which combine the desired structural characteristics from different natural products [23].

The goal of this work was to develop a virtual focused compound library of HIV-1 protease inhibitors from natural products fragments through de novo design. The focused library was compared with two virtual libraries of HIV-1 protease inhibitors developed from commercially available fragment libraries that were used as reference. The commercial reference libraries were 4,063 ChemDiv's fragments (enriched with sp ${ }^{3}$ carbons) [24], and 4,150 natural product fragments from Enamine [25]. The natural product fragments were built from the COlleCtion of Open NatUral producTs (COCONUT), the currently largest accessible database of natural products with more than 400,000 
non-redundant compounds [26]. We aim that the present work will contribute towards the research that leads to effective HIV treatments.

\section{Materials and Methods}

The virtual focused compound libraries of HIV-1 viral protease inhibitors from natural product fragments and two commercially available fragments libraries were developed using the protocol outlined in Figure 2.

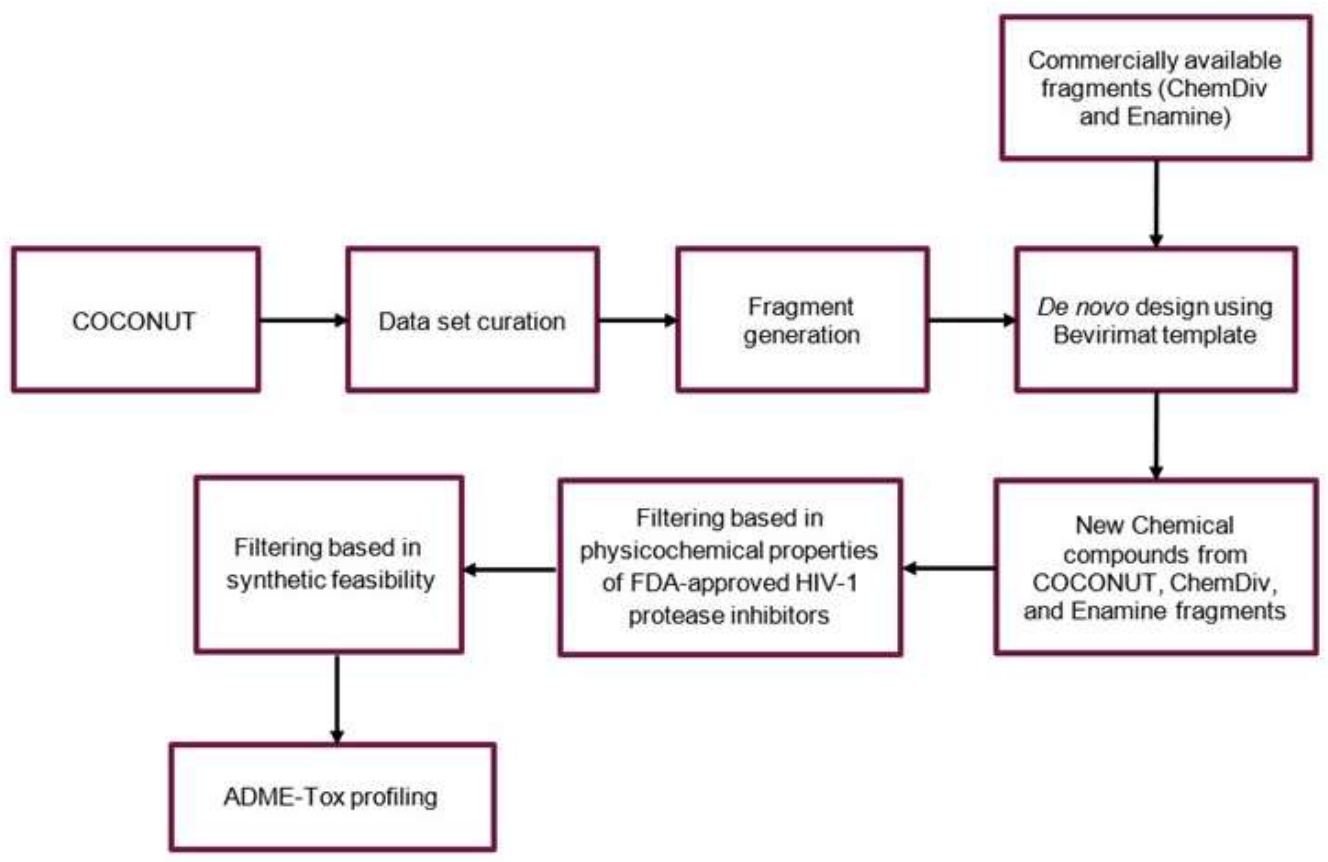

Figure 2: De novo design of the virtual focused compound libraries of HIV-1 viral protease inhibitors from natural product fragments (COCONUT) and commercially available fragments (ChemDiv and Enamine).

\subsection{Data set curation}

The preparation of compounds, encoded in Simplified Molecular Input Line System (SMILES) [27], was performed using the open-source cheminformatics toolkit RDKit [28], and tool MolVS [29]. Compounds with valence errors or any chemical element other than $\mathrm{H}, \mathrm{B}, \mathrm{C}, \mathrm{N}, \mathrm{O}, \mathrm{F}, \mathrm{Si}, \mathrm{P}, \mathrm{S}, \mathrm{Cl}, \mathrm{Se}, \mathrm{Br}$, and I were deleted. Stereochemistry information was removed because not all compounds in datasets have it defined. Compounds with multiple components were split, and the largest component was retained. The remaining compounds were neutralized and reionized to subsequently generate a canonical tautomer. Repeated compounds were deleted. To narrow down the search chemical space, physicochemical properties were computed: hydrogen bond donors (HBD), hydrogen bond acceptors (HBA), topological polar surface area (TPSA), number of rotatable bonds (RB), molecular weight (MW), and partition coefficient octanol/water (SlogP). Molecular compounds with the "rule of five" [30] and Veber [31] (MW $\leq 500, H B D \leq 5, H B A \leq 10$, $S \log P \leq 5, T P S A \leq 140, R B \leq 10$ ) were retained.

\subsection{Generation of unique fragments using retrosynthetic rules}

Fragment libraries were produced with the Retrosynthetic Combinatorial Analysis Procedure (RECAP) as implemented in RDKit. The RECAP algorithm [32] cleaves a molecule into fragments if this had any of the following bonds: amide, ester, amine, urea, 
ether, olefin, quaternary nitrogen, aromatic nitrogen-aliphatic carbon, lactam nitrogenaliphatic carbon, aromatics carbon-aromatic carbon, and sulphonamide.

\subsection{De novo design}

The new chemical structures were built based on the template previously proposed by Zhao et al. developed from the structure-activity relationship (SAR) analysis for the optimization of bevirimat (Figure 3), a compound derived from betulinic acid (Figure 4) [33]. Bevirimat [34,35] is a compound in clinical trials that targets the Gag polyprotein inhibiting the action of HIV protease at its the last cleavage event of the capsid protein and spacer peptide 1 (CA-SP1). [36,37]. The template proposed for building new chemical compounds related to bevirimat is shown in Figure 5.

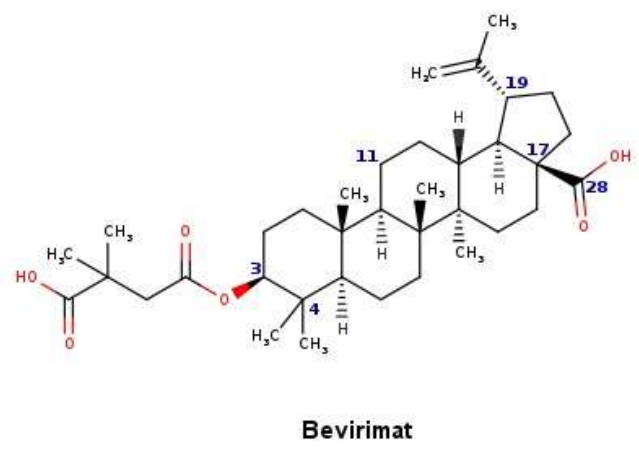

Figure 3: Chemical structure of bevirimat.

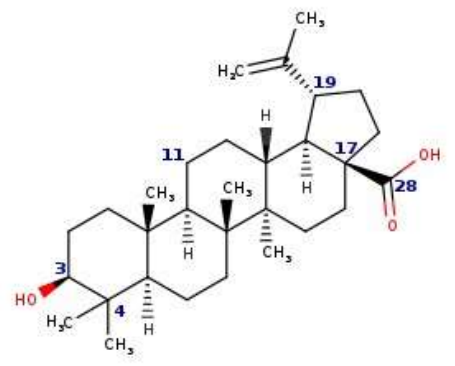

Betulinic acid

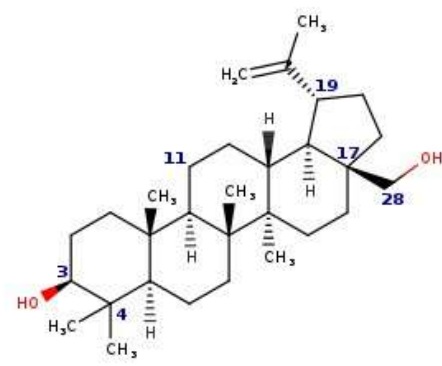

Betulin<smiles>C1CCC2C(C1)CCC1C3CCCC3CCC21</smiles>

Betulinic acid ring skeleton

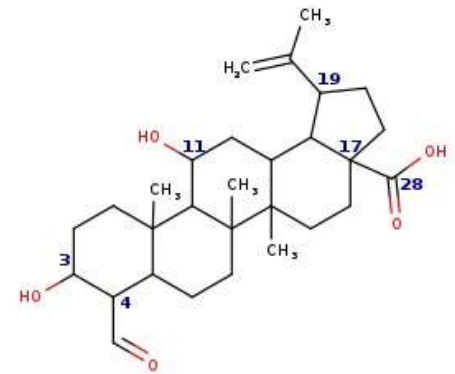

COCONUT's fragment

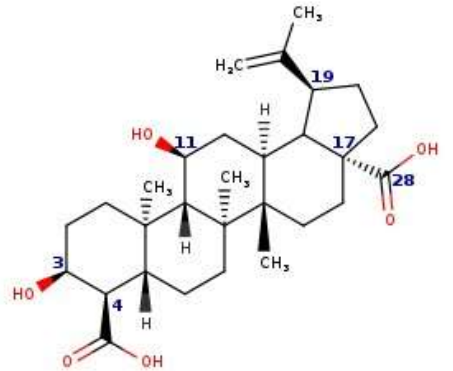

24-nor-3a,11a-dihydroxy-lup-20(29)-en-23,28-dioic acid

Figure 4: Chemical structures of betulinic acid, betulin, cyclic system skeleton derived from betulinic acid, COCONUT's fragment with betulinic acid ring skeleton derived from the 24-nor-3 $\alpha, 11 \alpha$-dihydroxy-lup-20(29)-en-23,28-dioic acid. 


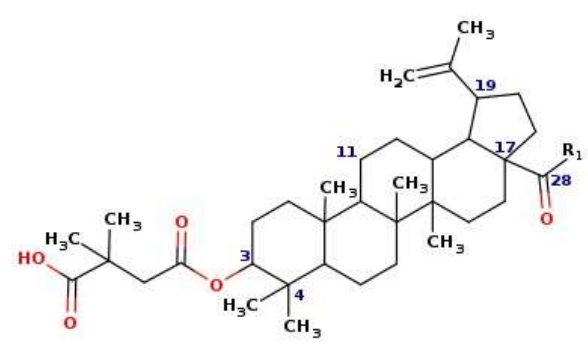

Betulinic acid ester

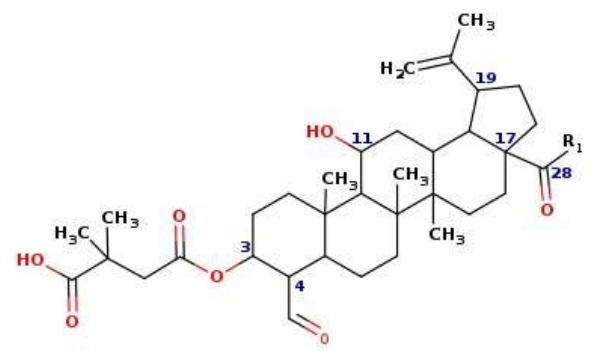

COCONUT's fragment

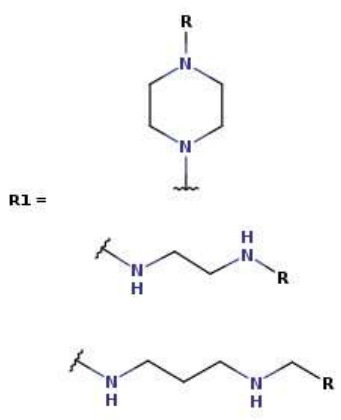

Figure 5: Template for building new chemical compounds similar to Bevirimat using the ester of betulinic acid to ChemDiv fragments and Enamine fragments, and the ester of COCONUT's fragment derived from 24-nor-3 $\alpha, 11 \alpha$-dihydroxy-lup-20(29)-en-23,28-dioic acid.

New molecules were generated using the Python programming language and the toolkit RDKit [28], following the protocol described for Saldívar-González et al. to enumerate chemical libraries [18]. We used COCONUT fragments with a cyclic system skeleton similar to betulinic acid, a hydroxyl group attached to carbon 3, and a carboxylic acid group attached to carbon 17, as shown in Figure 4. The COCONUT's fragment selected was derived from 24-nor-3 $\alpha, 11 \alpha$-dihydroxy-lup-20(29)-en-23,28-dioic acid (COCONUT ID: CNP0243494 or Reaxys ID: 6547020). Betulinic acid was used to build new chemical compounds from ChemDiv fragments and Enamine fragments because there were no fragments of cyclic system skeleton derived from betulinic acid or analogous triterpenes.

Chemical reactions were represented in SMIRKS, a hybrid notation of SMILES and SMARTS (SMILES Arbitrary Target Specification). Reaction 1, esterification, was made between triterpene alcohol and 2,2-dimethyl succinic acid using SMIRKS 1, as shown in Table 2. Reaction 2, amidation, was built from the carboxyl group attached to carbon 17 as shown in Figure 4 using fragments attached to piperazine, 1,3-diaminoethane, and 1,3-diaminopropane find in COCONUT fragments, ChemDiv fragments, and Enamine fragments. The SMIRKS 2.1-2.3 were used in reaction 2 and shown in Table 2. The compounds and fragments were selected using the functional groups in SMARTS notation described in Table 3. Newly generated chemical structures with valence errors were removed. Canonical SMILES were generated, and duplicate molecules were deleted. 
Table 2: SMIRKS used for building the new chemical compounds from natural products fragments.

Description

[\#6:1][\#6;A;X4:3]([\#6:2])[\#6:4]-[\#6:5]([\#8;A])=[O:6].[\#8:7]-[\#6:8]-1-[\#6:

SMIRKS 1 9]-[\#6:10]-[\#6:11]-2-[\#6:27](-[\#6:26]-[\#6:25]-[\#6:24]-3-[\#6:23]-4-[\#6:22][\#6:21][C:20]5([\#6:19]-[\#6:18]-[\#6:17]-[\#6:16]5-[\#6:15]-4-[\#6:14]-[\#6:13] -[\#6:12]-2-3)[\#6:29](-[\#8:31])=[O:30])-[\#6:28]-1>>[\#6:2][\#6;A;X4:3]([\#6: 1])[\#6:4]-[\#6:5](=[O:6])-[\#8:7]-[\#6:8]-1-[\#6:9]-[\#6:10]-[\#6:11]-2-[\#6:27]([\#6:26]-[\#6:25]-[\#6:24]-3-[\#6:23]-4-[\#6:22]-[\#6:21][C:20]5([\#6:19]-[\#6:18 ]-[\#6:17]-[\#6:16]5-[\#6:15]-4-[\#6:14]-[\#6:13]-[\#6:12]-2-3)[\#6:29](-[\#8:31]) $=[\mathrm{O}: 30])-[\# 6: 28]-1$

Reaction 2.1

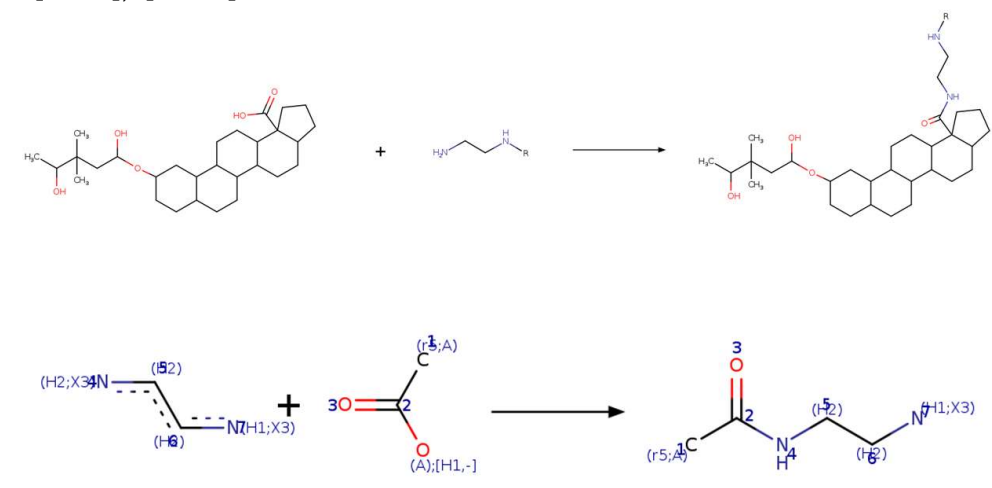

SMIRKS 2.1

[\#7;H1;X3:7][\#6H2:6][\#6;H2:5][\#7;H2;X3:4].[\#6;A;r5:1][\#6:2]([\#8;A;H1 $,-])=[\mathrm{O}: 3]>>[\# 6 ; \mathrm{A} ; \mathrm{r5}: 1][\# 6: 2](=[\mathrm{O}: 3])-[\# 7: 4]-[\# 6 ; \mathrm{H2}: 5]-[\# 6 ; \mathrm{H2}: 6]-[\# 7 ; \mathrm{H1}$ ;X3:7]

Reaction 2.2

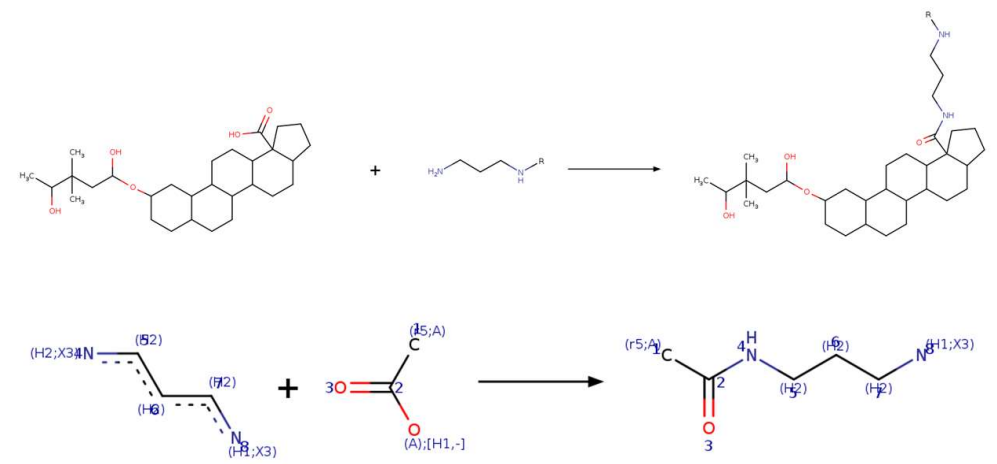

SMIRKS 2.2 [\#7;H1X3:8][\#6H2:7][\#6H2:6][\#6H2:5][\#7;H2X3:4].[\#6;A;r5:1][\#6:2]([\# 8;A;H1,-])=[O:3]>>[\#6;A;r5:1][\#6:2](=[O:3])-[\#7:4]-[\#6H2:5]-[\#6H2:6]-[ \#6H2:7]-[\#7;H1X3:8] 
Table 2 (continuation): SMIRKS used for building the new chemical compounds from natural products fragments.

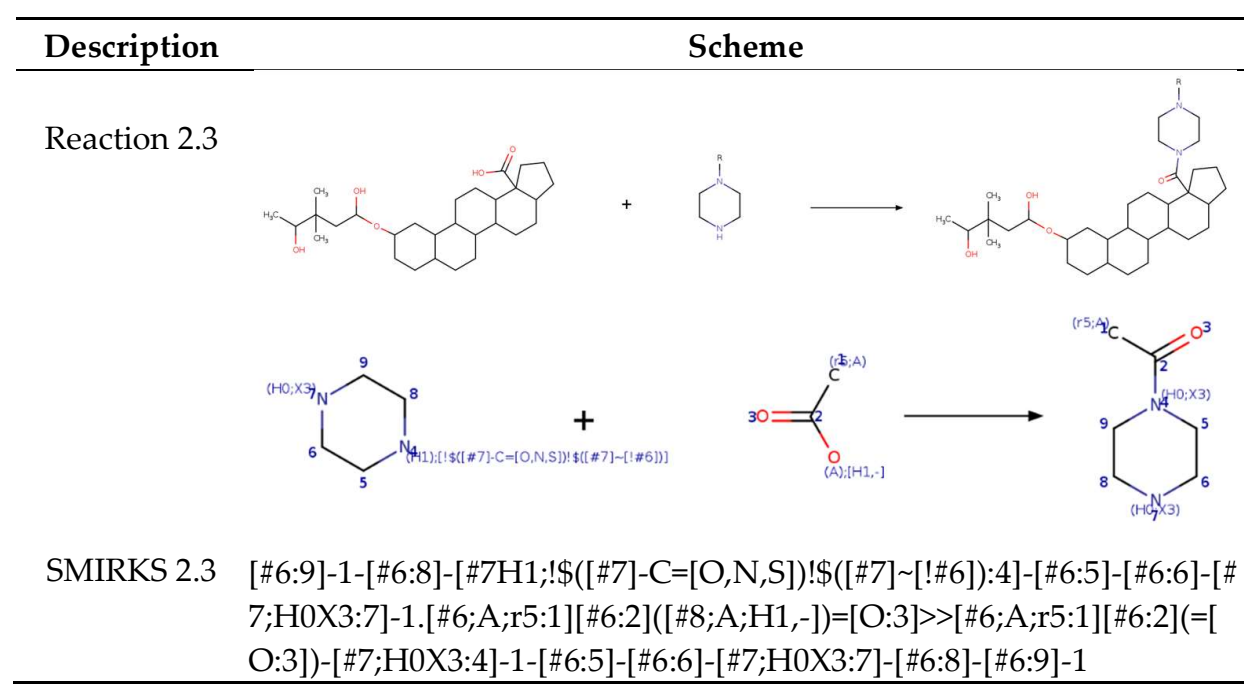

Table 3: Functional groups using SMARTS notation to filter fragments from natural products.

\begin{tabular}{|c|c|}
\hline Functional groups & SMARTS \\
\hline $\begin{array}{l}\text { Aliphatic alcohol } \\
\text { (cyclohexanol) }\end{array}$ & $\begin{array}{c}{[\# 8 ; H 1]-[\# 6]-1-[\# 6]-[\# 6]-[\# 6]-2-[\# 6](-[\# 6]-[\# 6]-[\# 6]-3-[\# 6]-4} \\
-[\# 6]-[\# 6] C 5([\# 6]-[\# 6]-[\# 6]-[\# 6] 5-[\# 6]-4-[\# 6]-[\# 6]-[\# 6]-2-3)[ \\
\# 6]([\# 8 ; H 1])=0)-[\# 6]-1\end{array}$ \\
\hline 2,2-dimethyl succinic acid & {$[\# 6] \mathrm{C}([\# 6])([\# 6]-[\# 6](-[\# 8])=\mathrm{O})[\# 6](-[\# 8])=\mathrm{O}$} \\
\hline piperazine & $\begin{array}{c}{[\# 6 ; \mathrm{H} 2 ; \mathrm{X} 4] 1-[\# 6 ; \mathrm{H} 2 ; \mathrm{X} 4][\# 7 ; \mathrm{X} 3 ; ! \mathrm{H} 1][\# 6 ; \mathrm{H} 2 ; \mathrm{X} 4]-[\# 6 ; \mathrm{H} 2 ; \mathrm{X} 4]} \\
{[\# 7 ; \mathrm{H} 1 ; \mathrm{X} 3] 1}\end{array}$ \\
\hline 1,2-diaminoethane & {$[\# 7 ; \mathrm{H} 1 ; \mathrm{X} 3][\# 6 ; \mathrm{H} 2 ; \mathrm{X} 4][\# 6 ; \mathrm{H} 2 ; \mathrm{X} 4][\# 7 ; \mathrm{H} 2 ; \mathrm{X} 3]$} \\
\hline 1,3-diaminopropane & {$[\# 7 ; \mathrm{H} 1 ; \mathrm{X} 3][\# 6 ; \mathrm{H} 2 ; \mathrm{X} 4][\# 6 ; \mathrm{H} 2 ; \mathrm{X} 4][\# 6 ; \mathrm{H} 2 ; \mathrm{X} 4][\# 7 ; \mathrm{H} 2 ; \mathrm{X} 3]$} \\
\hline $\begin{array}{l}\text { Cyclic system skeleton } \\
\text { derived from betulinic acid }\end{array}$ & $\begin{array}{l}{[\# 6] 1-[\# 6]-[\# 6]-[\# 6] 2-[\# 6](-[\# 6]-1)-[\# 6]-[\# 6]-[\# 6] 1-[\# 6]-2-[\#} \\
6]-[\# 6]-[\# 6] 2-[\# 6] 3-[\# 6]-[\# 6]-[\# 6]-[\# 6]-3-[\# 6]-[\# 6]-[\# 6]-1-2\end{array}$ \\
\hline
\end{tabular}

\subsection{Structural diversity and complexity}

The structural diversity of the new chemical compounds generated was evaluated to compute the median value of the distribution of the pairwise similarity values generated with the Tanimoto coefficient for Morgan fingerprint with radius 2 (Morgan2, 1024-bits) [38] and Molecular ACCes System (MACCS) Keys (166-bits) [39].

\subsection{Chemical space visualization}

The chemical space visualization was done using two methods, principal component analysis (PCA) based on physicochemical properties and the Tree MAP (TMAP) algorithm based on molecular fingerprints [40,41]. 
PCA is a linear dimensionality reduction technique to transform data with many dimensions into a lower dimensional space and preserve the different relationships between the data points as much as possible [42]. PCA was generated from six physicochemical properties (MW, HB, HBA, SlogP, TPSA, and RB).

TMAP allows the visual representation of many chemical compounds through the distance between the clusters and the cluster's detailed structure through Local Sensitive Hashing (LSH) forest data structure, enabling c-approximate k-nearest neighbors (k-NN). Morgan fingerprints for chemical compounds were encoded using the MinHash algorithm. The number of nearest-neighbors, $k=50$, and the factor used by the augmented query algorithm, $\mathrm{kc}=10$, were used to develop the TMAP graphs. Morgan fingerprints with radius 2 (Morgan2, 1024-bits) were generated to generate TMAP graphs [38]. Applications of TMAP for chemical space visualization of other compound data sets have been reported $[43,44]$.

\subsection{Filtering of the new chemical compounds generated}

To narrow down the search in chemical space and set the conditions for the newly generated compounds, physicochemical properties were computed for libraries generated and FDA-approved HIV-1 protease inhibitors (Table 1 and Figure 1). The maximum values of the physicochemical properties obtained from the HIV-1 protease inhibitors was $H B D \leq 6, H B A \leq 13, S \log P \leq 6.7, M W \leq 720.30$, TPSA $\leq 174.60$, and $R B \leq 17$ (Table 4). Molecules with at least four rules were retained. SlogP strictly must be complied. These sets of properties and values were used as a heuristic rule that is slightly less stringent than the Lipinski and Veber rules [30,31].

Table 4: Properties of pharmaceutical relevance of FDA-approved HIV-1 protease inhibitors.

\begin{tabular}{ccccccc}
\hline Parent molecule & SlogP & MW & HBD & HBA & TPSA & RB \\
\hline Amprenavir & 2.40 & 505.22 & 4 & 9 & 131.19 & 11 \\
Atazanavir & 4.21 & 704.39 & 5 & 13 & 171.22 & 14 \\
Darunavir & 2.38 & 547.24 & 4 & 10 & 140.42 & 11 \\
Fosamprenavir $^{\mathrm{a}}$ & 2.69 & 585.19 & 4 & 12 & 174.56 & 13 \\
Indinavir & 2.87 & 613.36 & 4 & 9 & 118.03 & 11 \\
Lopinavir & 4.33 & 628.36 & 4 & 9 & 120.00 & 15 \\
Nelfinavir & 4.75 & 567.31 & 4 & 7 & 101.90 & 9 \\
Ritonavir & 5.91 & 720.31 & 4 & 11 & 145.78 & 17 \\
Saquinavir & 3.09 & 670.38 & 6 & 11 & 166.75 & 12 \\
Tipranavir $^{\mathrm{a}}$ & 6.70 & 602.21 & 1 & 7 & 102.43 & 11 \\
Minimum $^{\mathrm{a}}$ & 2.40 & 505.20 & 1 & 7 & 101.90 & 9 \\
Maximum $^{\mathrm{a}}$ & 6.70 & 720.30 & 6 & 13 & 174.60 & 17 \\
\hline
\end{tabular}

a Maximum and minimum values for each property.

\subsection{Synthetic feasibility}

The complexity of the compounds generated was estimated using the synthetic accessibility score (SAscore) previously reported [45]. The SAscore implemented in this work is the difference between fragment score and complexity penalty. The fragment score captures common structural features in a large number of already synthesized molecules (934,046 representative molecules from the PubChem). Molecules are fragmented using extended connectivity fragments (ECFP_4\# fragments), and the fragment score is calculated as a sum of contributions of all fragments in the molecule divided by the number of fragments in the molecule. The fragment frequency is related to their synthetic accessibility, and hence easy-to-prepare substructures are present in molecules quite often. The complexity score is calculated as the sum of ring complexity (ring bridge atoms and spiro atoms), the number of stereocenters, large rings (ring size greater than eight, molecular complexity increases), and molecule size. The SAscore was calculated 
for the virtual focused libraries of HIV-1 viral protease inhibitors generated, and two reference data sets of FDA-Approved drugs, and FDA-Approved HIV-1 protease inhibitors [46]. The SAscore was calculated using the Python script published by Ertl and Schuffenhauer [45].

\subsection{ADME-Tox profiling}

Absorption, distribution, metabolism, excretion, and toxicity (ADME-Tox) properties of virtual focused libraries of HIV-1 viral protease inhibitors generated were calculated using the SwissADME server [47] and the pkCSM-pharmacokinetics server [48]. The ADME-Tox properties of FDA-Approved drugs were also computed as reference. The SwissADME server was used to compute descriptors associated with absorption and metabolism. The pkCSM-pharmacokinetics server was used to compute descriptors associated with absorption, distribution, excretion, and toxicity. The evaluation of descriptors related to ADME-Tox properties was computed as previously described [49]. The descriptors calculated were absorption broken down into solubility, Silico-IT LogSw; lipophilicity, consensus LogPo/w, and human intestinal absorption (HIA). The blood-brain barrier (BBB) permeability, P-glycoprotein substrate, P-glycoprotein I inhibitor, and P-glycoprotein II (take binary values: yes/no) for distribution. Inhibition of five main cytochrome enzymes (CYP-1A2, CYP-2C19, CYP-2C9, CYP-2D6, CYP-3A4) for metabolism (take binary values: yes/no). Total clearance $\log (\mathrm{mL} / \mathrm{min} / \mathrm{kg})$ to excretion. The hERG I/II inhibition, AMES toxicity, and hepatotoxicity to toxicity (take binary values: yes/no).

\section{Results and discussion}

As mentioned in the Introduction and Methods sections, new chemical compounds were built from two commercially available libraries: 4,063 ChemDiv fragments enriched with $\mathrm{sp}^{3}$ carbons, 4,160 Enamine natural products fragments, and 184,769 COCONUT fragments computationally generated in house. The total number of molecules generated were: 1,534 from COCONUT's fragments, 62 molecules from ChemDiv fragments, and 11 molecules from Enamine fragments. Fragments attached to 1,3-diaminopropane were not found in ChemDiv and Enamine's fragment collections. Similarly, fragments attached to 1,2-diaminoethane were not found in Enamine fragments.

\subsection{Structural diversity}

The median of similarity generated using Morgan2 and MACCS keys fingerprints are shown in brackets, respectively, and described in Table S1 in the supplementary material. FDA-Approved drugs $(0.096,0293)$ and FDA-Approved HIV-1 protease inhibitors $(0.253,0.558)$ were the most diverse data sets, following by compounds derived from COCONUT fragments $(0.605,0.817)$, ChemDiv fragments $(0.676,0.821)$, and Enamine fragments $(0.682,0.823)$. Compound computationally generated from fragment data sets were less diverse because these data sets are focused on bevirimant-like compounds.

\subsection{Chemical space visualization}

A visual representation of the chemical space based on physicochemical properties using PCA is shown in Figure 6. Principal component 1 recovered $73.6 \%$ of the variance, and principal component 2 recovered $21.2 \%$ of the variance. The accumulated variance recovered by the first two principal components represented in Figure 6 was $94.8 \%$. In this chemical space visualization, the compounds generated from the three fragment libraries are within the space of physicochemical properties of FDA-Approved drugs. Likewise, some compounds generated from COCONUT fragments had physicochemical properties similar to FDA-Approved HIV-1 protease.

To quantitatively define which data set is the most diverse, coverage space obtained by convex hull analysis derived from PCA was computed for each data set (Figure S1). 
The convex hull is defined as the minimum convex polygon so that the point set is either inside this polygon or at its border [50,51]. The convex hull area computed were for FDA-Approved drugs (737.59), HIV-1 protease inhibitors (1.11), compounds from COCONUT's fragments (3.18), compounds from ChemDiv's fragments (0.79), and compounds from Enamine fragments (0.18). The outcome of this analysis was similar to the results of the structural diversity analysis based on fingerprints (section 3.1): reference data sets were more diverse than the new chemical compounds generated from fragments data sets. The new chemical compounds derived from COCONUT fragments were the most diverse, followed by new chemical compounds derived from ChemDiv and Enamine fragments.

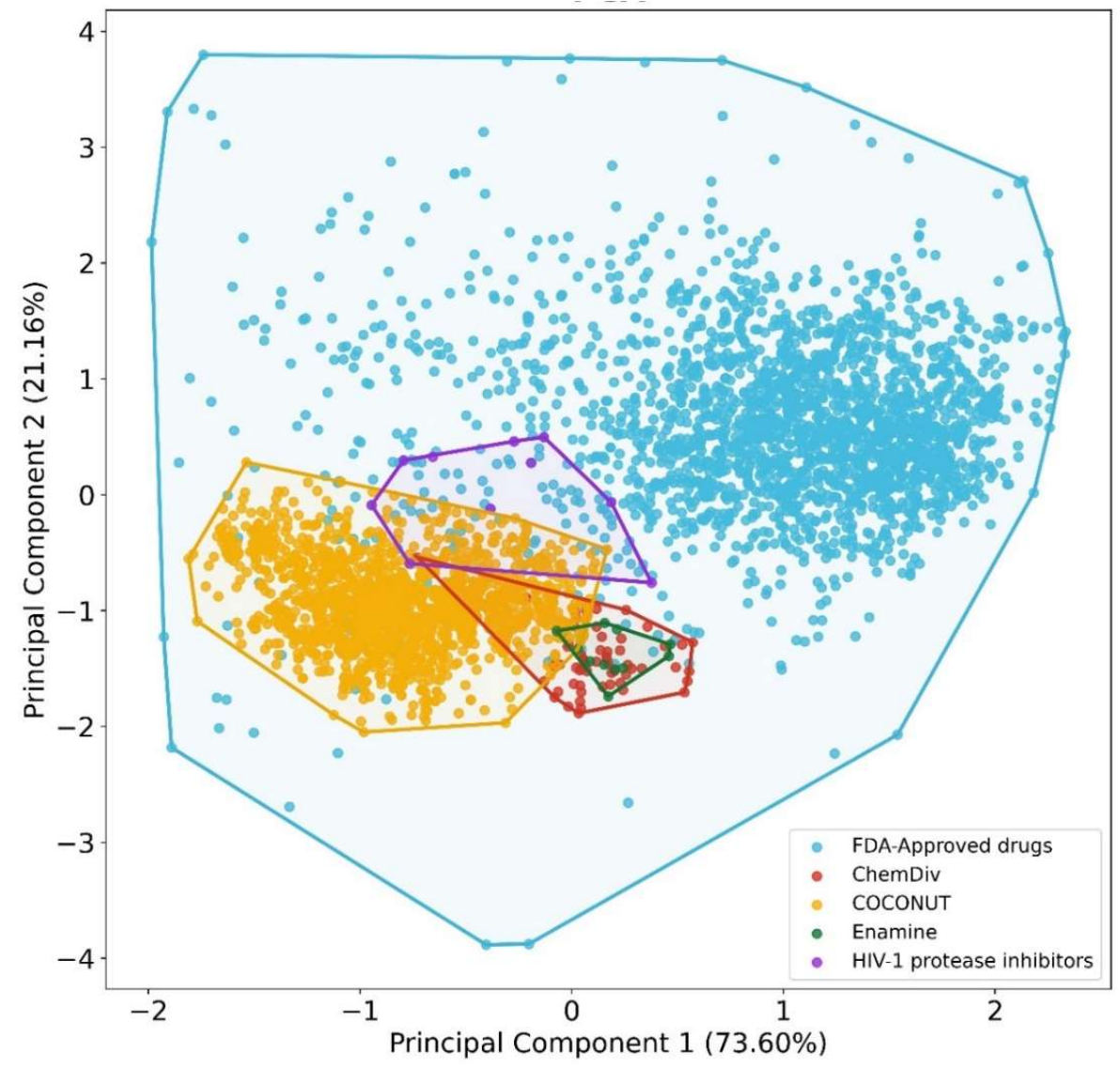

Figure 6: Chemical space visualization of the virtual focused compound library of HIV-1 viral protease inhibitors from natural product fragments and two compound reference libraries using PCA based on physicochemical properties. Compound reference libraries represented in colors: FDA-Approved drugs (blue) and FDA-Approved HIV-1 protease inhibitors (purple). Likewise. for new chemical compounds generated from COCONUT (orange), ChemDiv (red), and Enamine (green) fragment libraries.

The visual representation of the chemical space based on molecular fingerprint using the TMAP algorithm is shown in Figure 7. An interactive version of the TMAP is available at https://figshare.com/s/ceb58d58e8f5585ce67e. The chemical structures of new chemical compounds generated were very different in comparison with FDA-Approved drugs and FDA-HIV-1 protease inhibitors. The chemical structures of the new compounds generated from ChemDiv and Enamine fragments were very similar compared to compounds derived from COCONUT fragments. In some cases, the chemical structures of compounds generated from COCONUT's fragments were very similar to some FDA-Approved drugs, for instance, palbociclib and pipecuronium. In these cases where 
there are not commercially available fragments like COCONUT's fragments could be used palbociclib and pipecuronium.

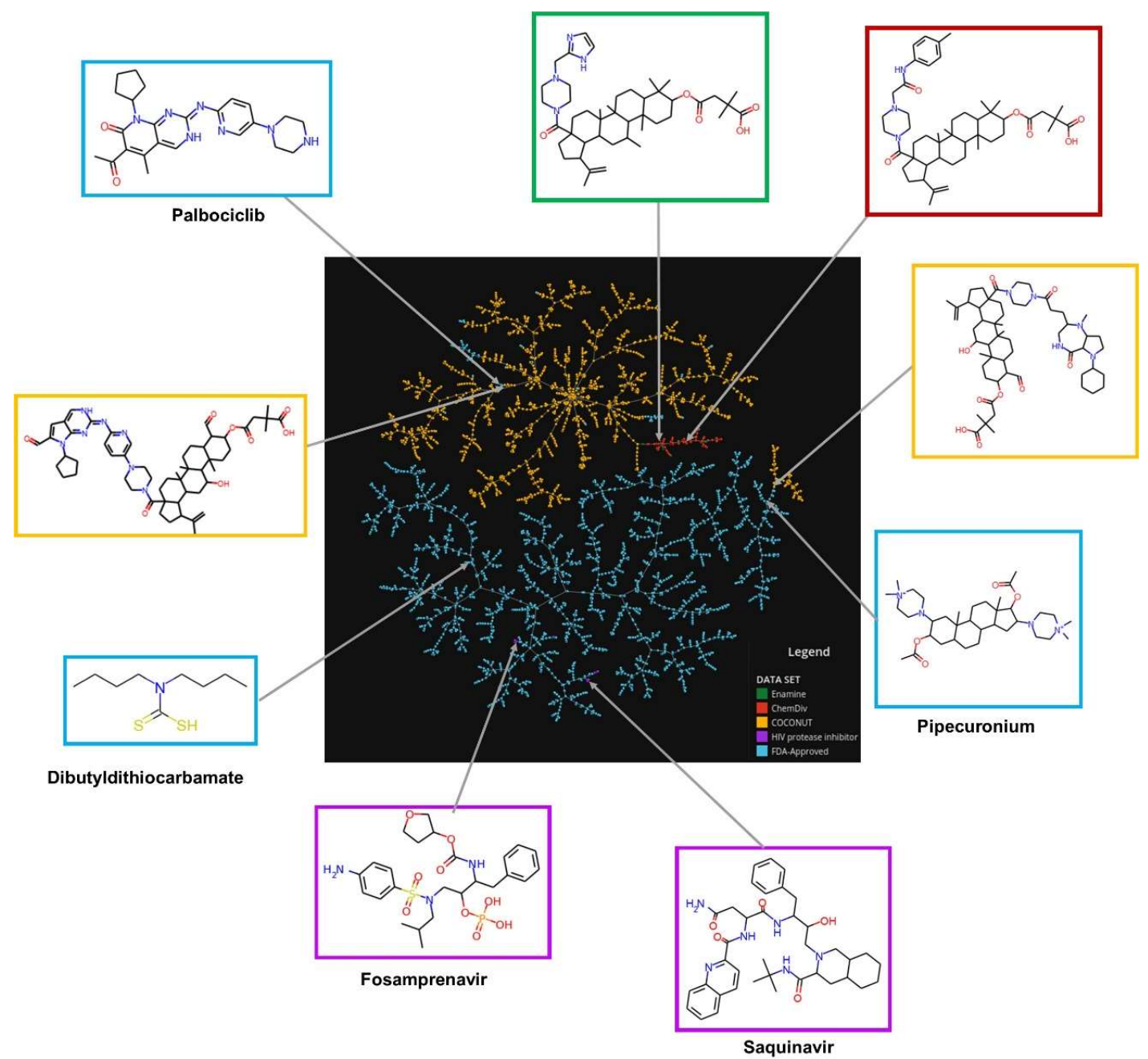

Figure 7: Chemical space visualization of the virtual focused compound library of HIV-1 viral protease inhibitors from natural product fragments and two compound reference libraries using TMAP based on molecular fingerprints. Compounds reference libraries represented in colors: FDA-Approved drugs (blue), and FDA-Approved HIV-1 protease inhibitors (purple). Likewise, for new chemical compounds generated from COCONUT (orange), ChemDiv (red), and Enamine (green) fragment libraries. The interactive version is available at https://figshare.com/s/ceb58d58e8f5585ce67e.

\subsection{Compound filterin based on physicochemical properties}

Figure 8 shows box-whisker plots of physicochemical properties after applying the empirical rules proposed (section 2.6). The summary of descriptive statistics is shown in Table S2-S7 in the supplementary material. 352 compounds generated from COCONUT fragments $(20 \%)$ and 1 compound generated from ChemDiv fragments were retained $(2 \%)$, and compounds generated from Enamine fragments were not retained $(0 \%)$. Based on the properties distribution shown in the box-whisker plots, the physicochemical properties of compounds generated from COCONUT fragments, ChemDiv fragments, and Enamine fragments were different regarding FDA-Approved HIV-1 protease inhibitors and FDA-Approved drugs.

The physicochemical properties calculated for data sets were: $\operatorname{Slog} \mathrm{P} \leq 12.94$, MW $\leq 1201.84, \mathrm{RB} \leq 20$, TPSA $\leq 286.50, \mathrm{HBA} \leq 23, \mathrm{HBD} \leq 15$ for FDA-Approved drugs; 
SlogP $\leq 6.70, \mathrm{MW} \leq 720.31, \mathrm{RB} \leq 17, \mathrm{TPSA} \leq 174.56, \mathrm{HBA} \leq 13, \mathrm{HBD} \leq 6$ for FDA-Approved HIV-1 protease inhibitors; SlogP $\leq 6.69, \mathrm{MW} \leq 998.63, \mathrm{RB} \leq 15, \mathrm{TPSA} \leq 198.54, \mathrm{HBA} \leq 13$, $\mathrm{HBD} \leq 7$ for compounds generated from COCONUT fragments, and SlogP=6.4, $\mathrm{MW}=737.47, \mathrm{RB}=10$, TPSA=187.47, $\mathrm{HBA}=12, \mathrm{HBD}=5$ for the compound generated from ChemDivs fragments. The SlogP, RB, and HBA values of compounds generated from COCONUT fragments and ChemDiv fragments were less than FDA-Approved HIV-1 protease inhibitors. HBA values were equal or less than FDA-Approved HIV-1 protease inhibitors. The SlogP values of compounds derived from Enamine fragments were larger than FDA-Approved HIV-1 protease inhibitors as shown in Figure S2; accordingly, no compound was retained. The MW, TPSA, and HBD values of compounds generated from COCONUT fragments were larger than for FDA-Approved HIV-1 protease inhibitors and less than for FDA-Approved drugs. As mentioned above Ganesan [52], natural products that violate the Lipinsky rules remain largely compliant in terms of $\log \mathrm{P}$ and HBD. He considers that "nature has learned to maintain low hydrophobicity and intermolecular H-bond donating potential when it needs to make biologically active compounds with high molecular weight and a large number of rotatable bonds". In drugs, the molecules that exceed HBD 5 or HBA 10 the majority are natural product-related [53].
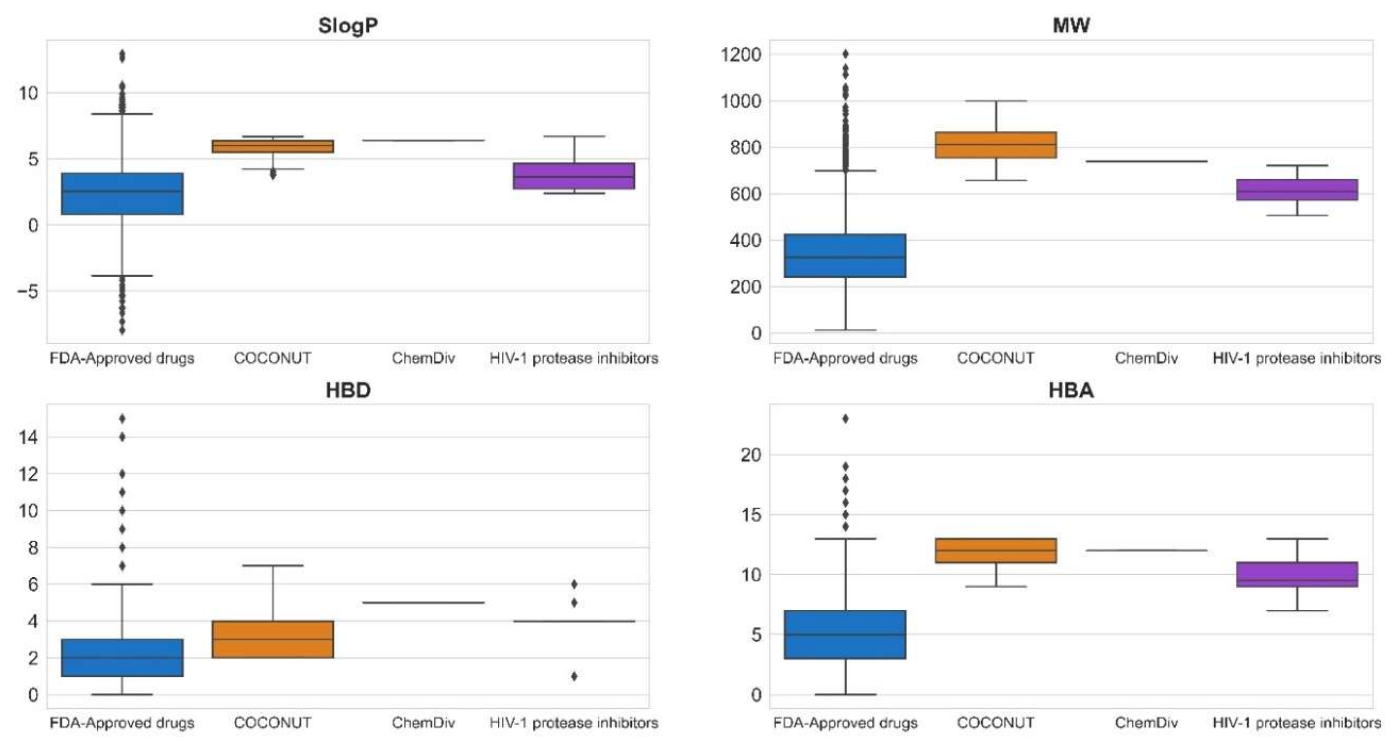
RB
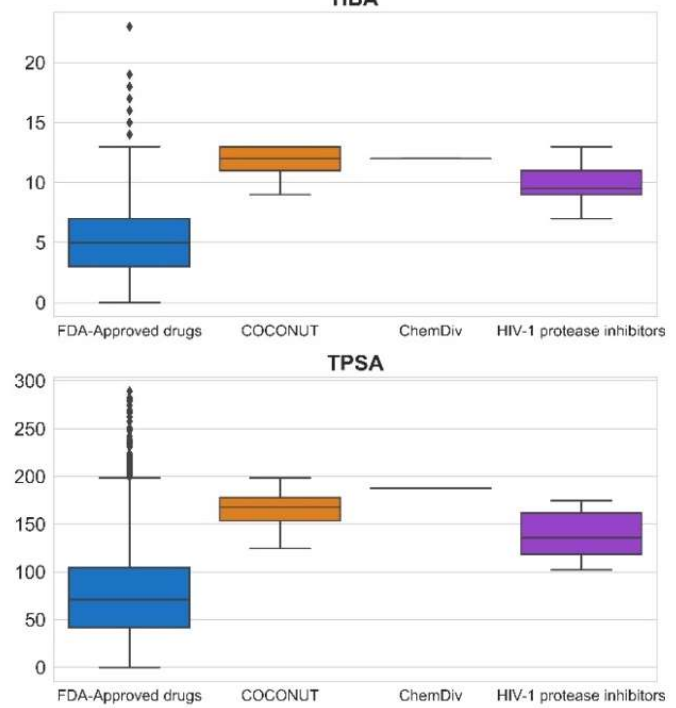

FDA-Approved drugs COCONUT ChemDiv HIV-1 protease inhibitor

Figure 8: Box-whisker plots of physicochemical properties of FDA-Approved drugs (blue), FDA-Approved HIV-1 protease inhibitors (purple), and new chemical compounds generated from COCONUT (orange) and ChemDiv (red) fragment libraries after applying physicochemical properties filtering.

\subsection{Filtering based on synthetic feasibility}

The synthetic feasibility was computed for FDA-Approved drugs, FDA-Approved HIV-1 protease inhibitors, and compounds generated from COCONUT and ChemDiv fragments with physicochemical properties like FDA-Approved HIV-1 protease inhibitors. Figure 9 summarizes the results of synthetic feasibility. Molecules with a low 
SAscore value $<6$ are easily synthetically accessible [45]. 97\% FDA-Approved drugs had SAscore $<6$, and FDA-Approved HIV-1 protease inhibitors had SAscore $\leq 4.24$. Similarly, $75 \%$ of compounds generated from COCONUT fragments had SAscore $\leq 6.03$ and the compound generated from ChemDiv had SAscore=5.54. Although, compounds generated from COCONUT fragments had $5.50 \leq S A s c o r e \leq 6.03$, still in recommended range so that can be synthetically accessible; moreover, the high SAscore in compounds generated regarding FDA Approved HIV-1 protease inhibitors was influenced by the ten stereocenters of betulinic acid and 24-nor-3 $\alpha, 11 \alpha$-dihydroxy-lup-20(29)-en-23,28-dioic acid. Considering that these stereocenters do not have to be generated within the organic synthesis, the SAscore value would be lower.

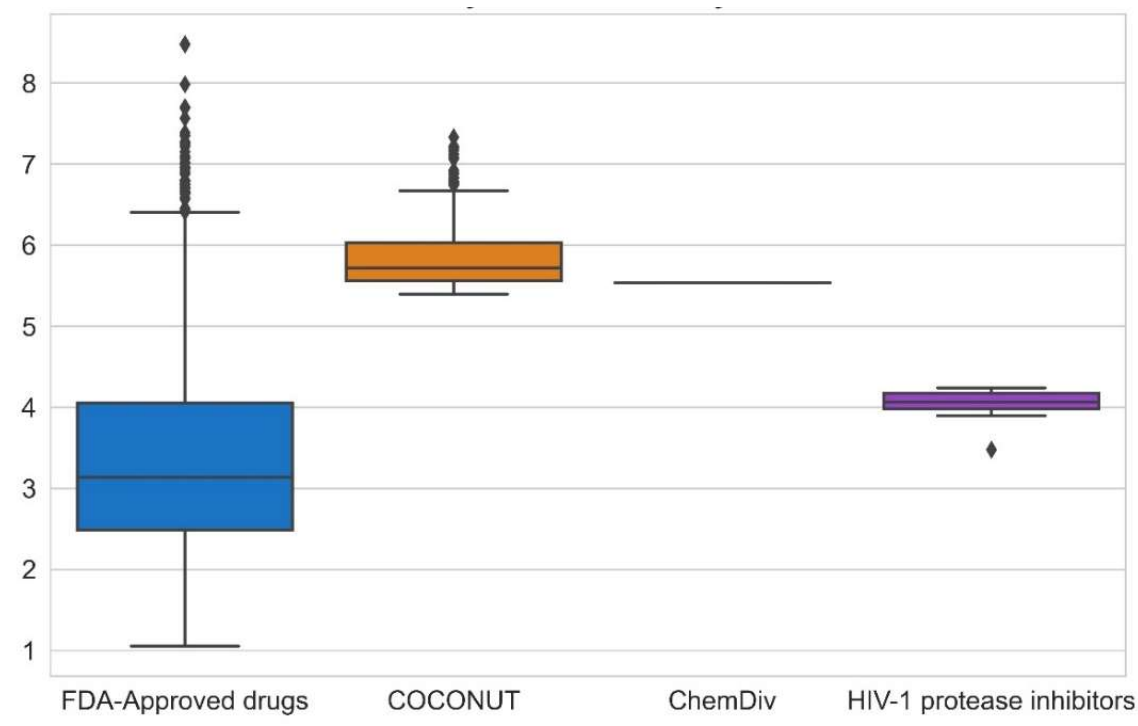

Figure 9: Box-whisker plot of synthetic feasibility calculated for FDA-Approved drugs (blue), FDA-Approved HIV-1 protease inhibitors (purple), and new chemical compounds generated from COCONUT fragments (orange) and ChemDiv (red) fragments with physicochemical properties like FDA-Approved HIV-1 protease inhibitors.

\subsection{ADME-Tox profiling}

The ADME-Tox profiling was computed for 251 compounds generated from COCONUT fragments and 1 compound generated from ChemDiv fragments with physicochemical properties like FDA-Approved HIV-1 protease inhibitors and estimated as easy synthesizable (i.e., SAscore $\leq 6)$. Similarly, ADME-Tox profiling was computed for FDA-Approved drugs and FDA-Approved HIV-1 protease inhibitors.

\subsubsection{Absorption}

Solubility, lipophilicity, and HIA are summarized in Figure 10 and Table S9-11 in the supplementary material. Solubility was expressed by Silicos-IT LogSw and lipophilicity was expressed by consensus $\log \mathrm{P}$. Silicos-IT LogSw and consensus $\log \mathrm{P}$ were computed with the SwissADME server. Percentage of HIA was computed with the pkCSM-pharmacokinetics server.

Median values for solubility, lipophilicity, and HIA are described below. FDA-Approved drugs had consensus $\log \mathrm{P}=2.36$, Silicos-IT $\log \mathrm{S}_{w}=-4.34$, HIA=90.6\%. FDA-Approved HIV-1 protease inhibitors had consensus LogP $=3.50$, Silicos-IT $\log \mathrm{Sw}_{\mathrm{w}}=-8.49$, HIA=64.4\%. Compounds derived from COCONUT and ChemDiv had consensus $\log \mathrm{P}=4.70$ and Silicos-IT $\log \mathrm{S}_{w}=-6.45, \mathrm{HIA}=67.9 \%$.

New drug candidates have poor water solubility, and it is often the result of highly lipophilic compounds. $\log \mathrm{P}<2$, the crystal lattice becomes the main determining factor for solubility. $\log \mathrm{P}$ values above 2, the lipophilicity is the main factor [54]. 
FDA-Approved HIV-1 protease inhibitors were highly soluble, followed by compounds derived from COCONUT and ChemDiv fragments, both had Log P>2; in this case, solubility is strongly influenced by lipophilicity. Contrary to FDA-Approved drugs that had Log P close to 2 and were less soluble, solubility mainly depends on the crystal lattice. Compounds derived from COCONUT and ChemDiv fragments had higher HIA in comparison to FDA-Approved HIV-1 protease inhibitors.
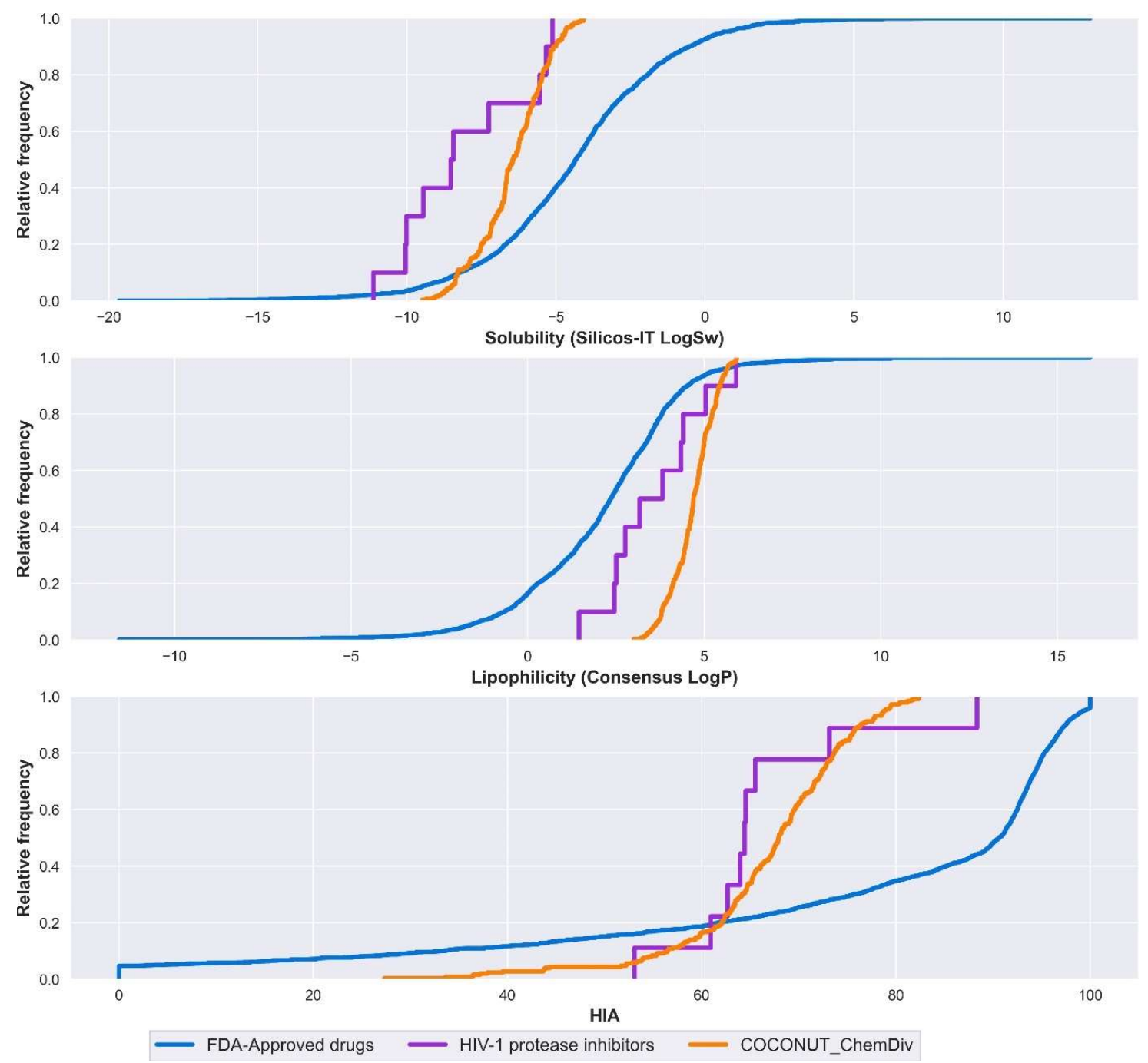

Figure 10: Distribution curve of solubility, lipophilicity, and HIA. Colors represent compounds: new chemical compounds generated from COCONUT fragments and ChemDiv fragments with physicochemical properties like FDA-Approved HIV-1 protease inhibitors and easily synthetically accessible (orange), FDA-Approved drugs (blue), FDA-Approved HIV-1 protease inhibitors (purple). Solubility is expressed in the percentage of Silicos-IT LogSw, and lipophilicity is expressed in the percentage of consensus LogP.

\subsubsection{Distribution}

The relative frequency of $\mathrm{BBB}$ permeability is described in Figure 11. The median value of BBB permeability was -0.38 for FDA-Approved drugs; -1.21 for compounds generated from COCONUT and ChemDiv fragments, and -1.25 for FDA-Approved HIV-1 protease inhibitors. Compounds generated from COCONUT and ChemDiv fragments had similar BBB permeability.

The percentage of compounds that are P-glycoprotein substrate, P-glycoprotein I inhibitor, and P-glycoprotein II inhibitor were summarized in Figure 12 and Table S13 in 
the supplementary material. All FDA-Approved HIV-1 protease inhibitors and 96\% of compounds generated from COCONUT and ChemDiv fragments were P-glycoprotein substrates. Similarly, $66.67 \%$ of HIV-1 Approved protease inhibitors and $82.9 \%$ of compounds generated from COCONUT and ChemDiv fragments were P-glycoprotein II inhibitors. Whereas no compounds generated from COCONUT and ChemDiv fragments were P-glycoprotein I inhibitors, against 100\% FDA-Approved HIV-1 proteases inhibitors were P-glycoprotein I inhibitors.

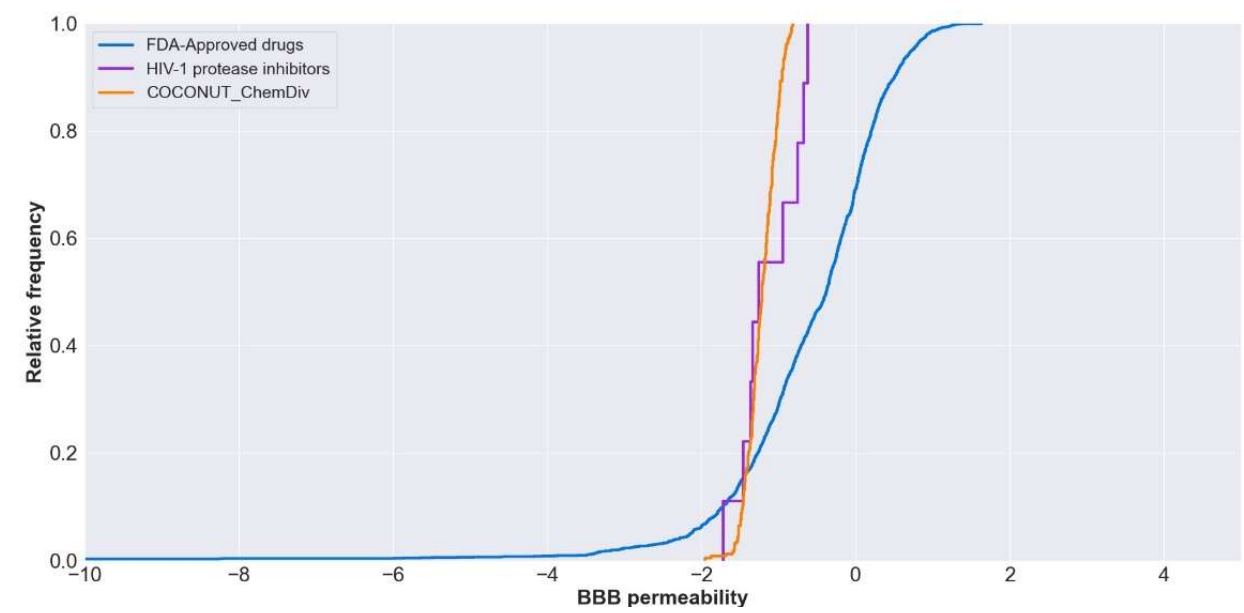

Figure 11: Distribution curve of BBB permeability. Colors represent compounds: new chemical compounds generated from COCONUT fragments and ChemDiv fragments with physicochemical properties like FDA-Approved HIV-1 protease inhibitors and easily synthetically accessible (orange), FDA-Approved drugs (blue), FDA-Approved HIV-1 protease inhibitors (purple). The BBB permeability of FDA-Approved drugs was between -34 and 2 . 

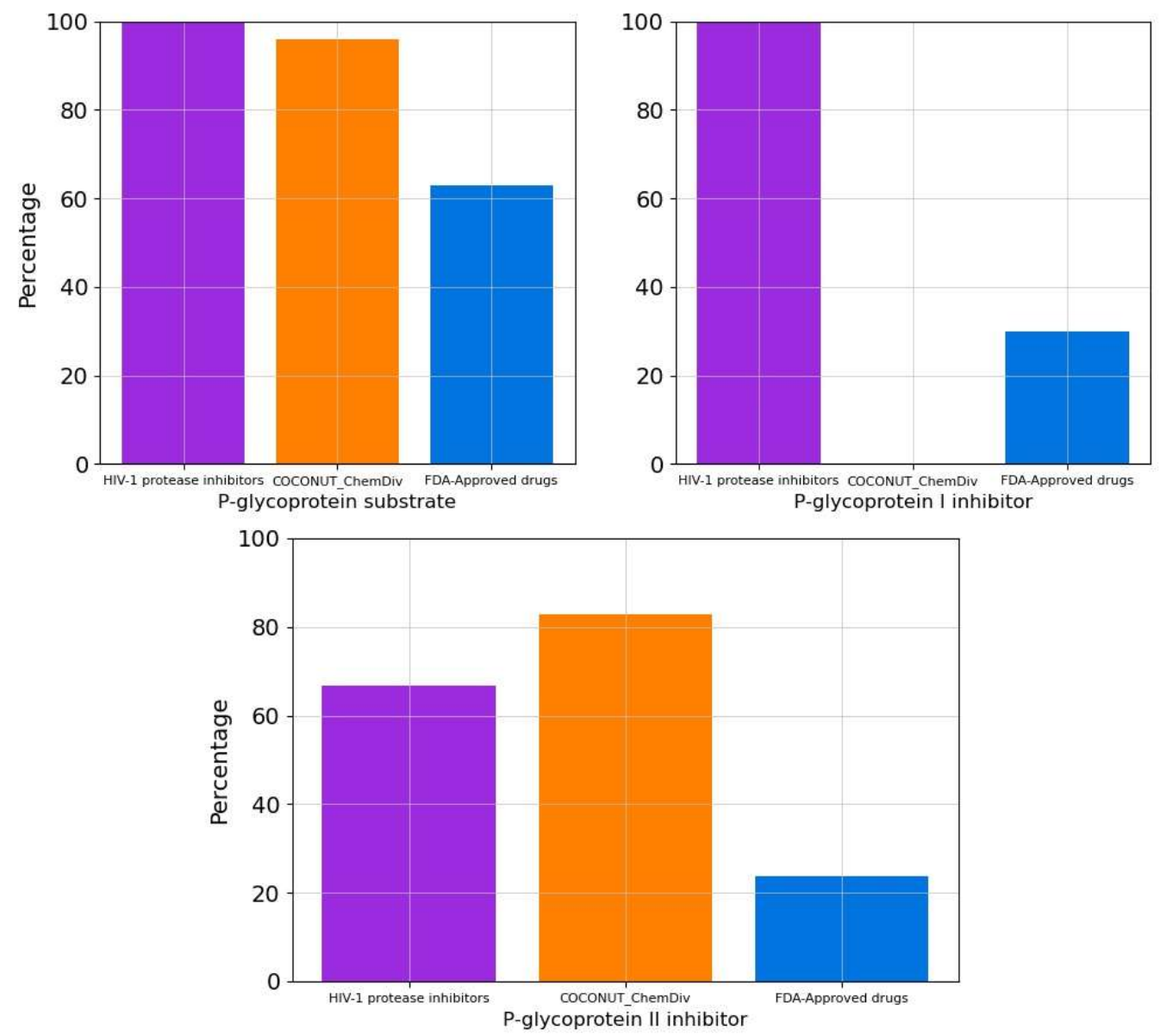

Figure 12: Percentage of compounds that are P-glycoprotein substrate, P-glycoprotein I inhibitor, and P-glycoprotein II inhibitor. Colors represent compounds: new chemical compounds generated from COCONUT fragments and ChemDiv fragments with physicochemical properties like FDA-Approved HIV-1 protease inhibitors and easily synthetically accessible (orange), FDA-Approved drugs (blue), FDA-Approved HIV-1 protease inhibitors (purple).

\subsubsection{Metabolism}

The percentage of compounds CYP1A2, CYP2C19, CYP2C9, CYP2D6, and CYP3A4 inhibitors is described in Figure 13 and Table S14 in the supplementary material. No compounds generated from COCONUT and ChemDiv fragments were CYP1A2, CYP2C19, CYP2C9, CYP2D6, and CYP3A4 inhibitors. FDA-Approved HIV-1 inhibitors were not CYP1A2 and CYP2D6 inhibitors similar to compounds generated from COCONUT and ChemDiv fragments. Whereas for FDA-Approved HIV-1protease inhibitors, $89 \%$ were CYP3A4 inhibitors, and $33 \%$ were CYP2C19 and CYP2C9 inhibitors. 

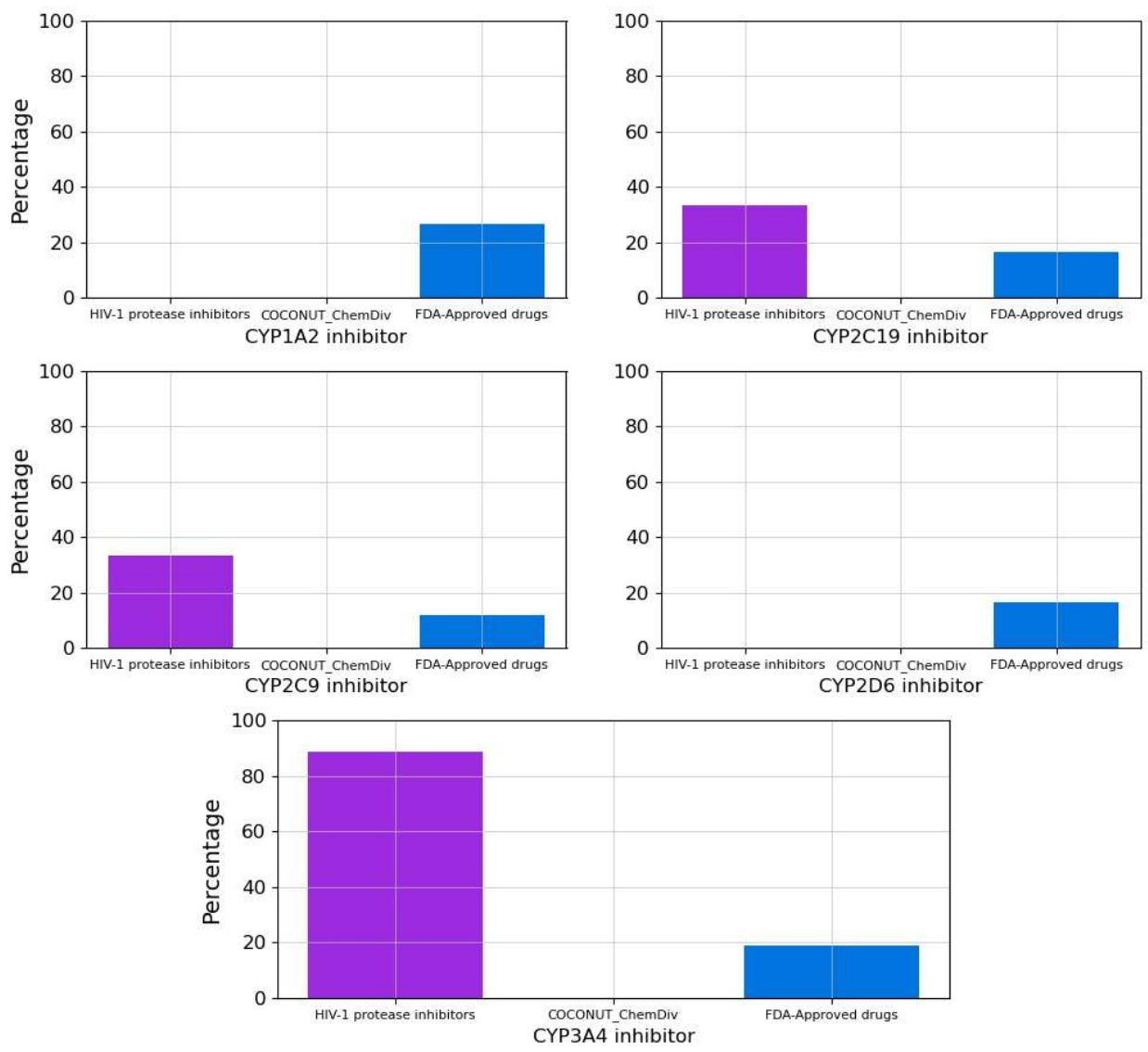

Figure 13: Percentage of compounds that inhibit the main cytochromes, CYP1A2, CYP2C19, CYP2C9, CYP2D6, CYP3A4. Colors represent compounds: new chemical compounds generated from COCONUT fragments and ChemDiv fragments with physicochemical properties like FDA-Approved HIV-1 protease inhibitors and easily synthetically accessible (orange), FDA-Approved drugs (blue), FDA-Approved HIV-1 protease inhibitors (purple).

\subsubsection{Excretion}

Clearance quantitates the irreversible removal of a drug from the measured matrix, generally, blood or plasma [55]. The total clearance logarithm expressed in units of $(\mathrm{mL} / \mathrm{min} / \mathrm{Kg})$ is shown in Figure 14. The summary of descriptive statistics is shown in Table S15 in the supplementary material. The median values of the total clearance logarithm were 0.591 for FDA-Approved drugs; 0.494 for FDA-Approved HIV-1 protease inhibitors, and -0.618 for compounds derived from COCONUT and ChemDiv fragments. The total clearance of FDA-Approved HIV-1 protease inhibitors (0.20 total clearance $\leq 0.94)$ was similar to $75 \%$ FDA-Approved drugs ( $0.27 \leq$ total clearance $\leq 0.85)$. Whereas the total clearance of compounds generated from COCONUT and ChemDiv fragments $(-1.34 \leq$ total clearance $\leq 0.13)$ was similar to $25 \%$ FDA-Approved drugs $(-13.94 \leq$ total clearance $\leq 0.27)$. The total clearance of compounds derived from COCONUT and ChemDiv fragments and FDA-Approved HIV-1 inhibitors were different. 


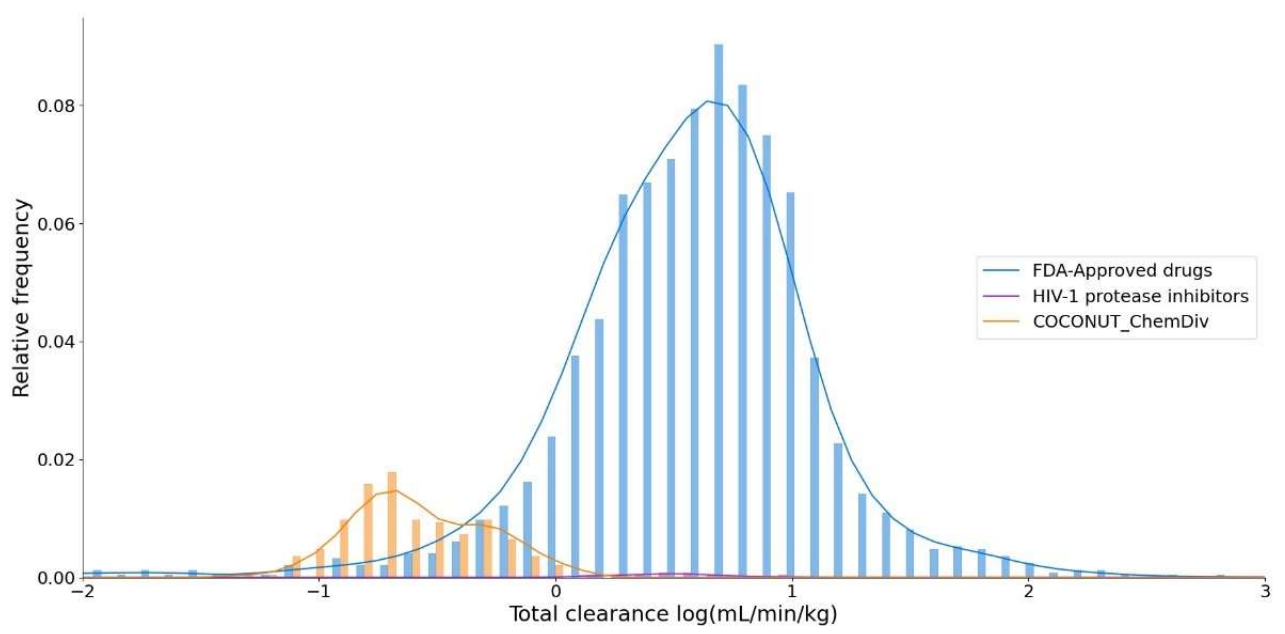

Figure 14: Distribution curve of the total clearance. Colors represent compounds: new chemical compounds generated from COCONUT fragments and ChemDiv fragments with physicochemical properties like FDA-Approved HIV-1 protease inhibitors and easily synthetically accessible (orange), FDA-Approved drugs (blue), FDA-Approved HIV-1 protease inhibitors (purple).

\subsubsection{Toxicity}

Percentage of compounds from data sets that are hERG I inhibitor, hERG II inhibitor, hepatotoxicants (hepatotoxicity), and carcinogens (positive in AMES test) were described in Figure 15 and Table S16 in the supplementary material. FDA-Approved HIV-1 protease inhibitors and compounds generated from COCONUT and ChemDiv fragments were not carcinogens. However, $77.22 \%$ of compounds derived from COCONUT and ChemDiv fragments were hepatotoxicants, lower than FDA-Approved HIV-1 protease inhibitors (100\%), and higher than FDA-Approved drugs (47.42\%). $100 \%$ and $98.81 \%$ of compounds generated from COCONUT and ChemDiv fragments were not hERG I/II inhibitors, respectively.
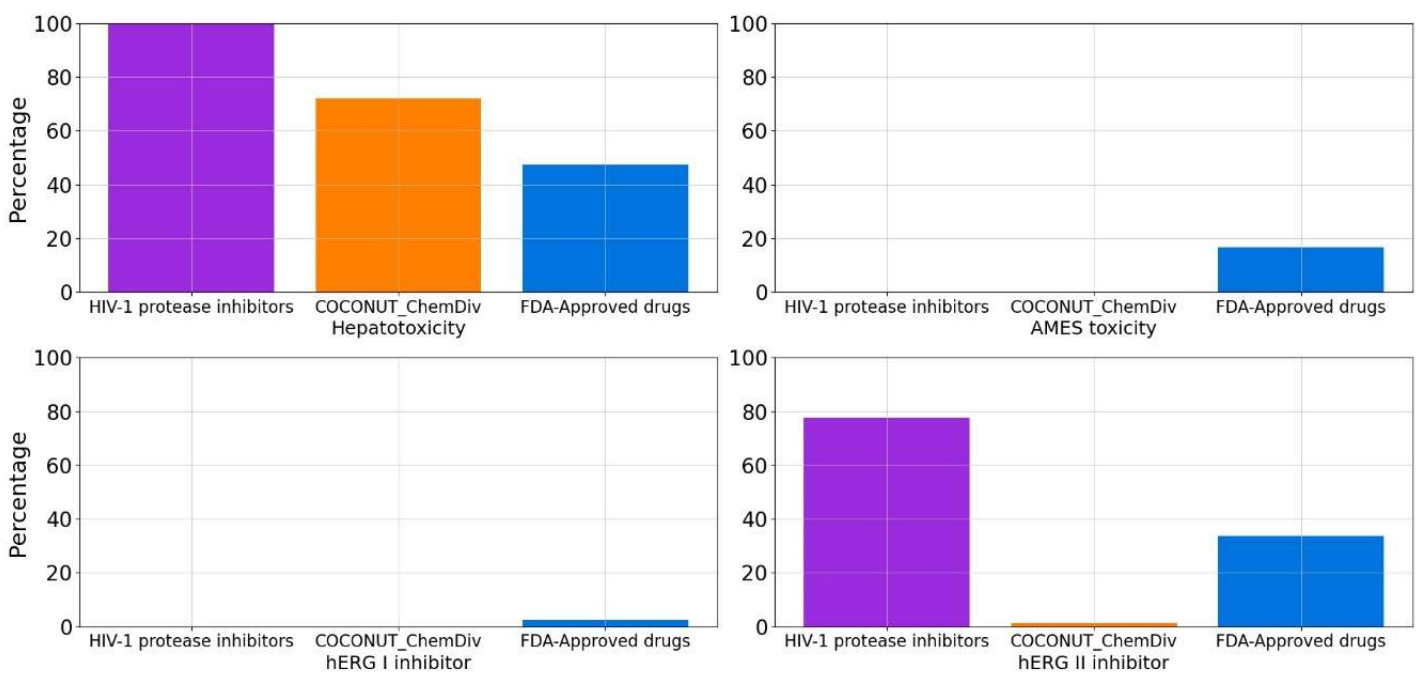

Figure 15: Percentage of compounds that are hERG I inhibitor, hERG II inhibitor, hepatotoxicity, and toxicity in AMES test in silico. Colors represent compounds: new chemical compounds generated from COCONUT fragments and ChemDiv fragments with physicochemical properties like FDA-Approved HIV-1 protease inhibitors and easily synthetically accessible (orange), FDA-Approved drugs (blue), FDA-Approved HIV-1 protease inhibitors (purple).

\section{Conclusions}


We developed an HIV-1 virtual focused library using de novo design based on enumerated libraries of compounds from fragment libraries. The fragments library in-house was built from the COCONUT database, the currently largest accessible database of natural products. Using bevirimat as template, 251 out of 1,534 compounds generated from COCONUT fragments, had physicochemical properties like FDA-Approved HIV-1 protease inhibitors and were estimated as easy synthesizable.

Compounds generated from COCONUT fragments were more diverse than compounds generated from ChemDiv and Enamine fragments, based on chemical structure and physicochemical properties. Visual representation of the chemical space based on TMAP showed that some compounds generated from COCONUT fragments had chemical structures similar to FDA-Approved drugs such as palbociclib and pipecuronium.

ADME/Tox profiling showed that compounds generated from COCONUT fragments had adsorption (solubility and lipophilicity) and distribution (BBB permeability, P-glycoprotein substrate, and P-glycoprotein II inhibitor) similar to FDA-Approved HIV-1 protease inhibitors. Concerning estimations of metabolism, no compounds generated from COCONUT fragments were CYP1A2, CYP2C19, CYP2C9, CYP2D6, and CYP3A4 inhibitors. As per excretion, the total clearance of compounds derived from COCONUT fragments and FDA-Approved HIV-1 inhibitors were different, but similar to FDA-Approved drugs. Compounds derived from COCONUT fragments were predicted to be no inhibitors of hERG I/II, like $97.7 \%$ and $66.4 \%$ of FDA-Approved drugs, respectively. Compounds derived from COCONUT fragments were predicted to be no carcinogens.

The 251 compounds derived from COCONUT fragments with physicochemical properties like FDA-Approved HIV-1 protease inhibitors, estimated as easy synthesizable, and good ADME/Tox profiling can be used in future analysis such as virtual screening to select candidates to test in biological assays.

The protocol presented in this work is general and can be used to build other chemical compounds like bevirimat or other maturation inhibitors of HIV-protease. This can be achieved from the SMARTS and SMIRKS proposed to filter functional groups and build new chemical compounds.

Supplementary Materials: The following are available online at www.mdpi.com/xxx/s1, Figure S1: Convex hull area from PCA based on physicochemical properties of new chemical compounds generated and two compound reference libraries. Table S1: Summary of fingerprint-based structural diversity of new chemical compounds generated from COCONUT, ChemDiv, and Enamine fragments, and two compound reference libraries. Figure S2: Box-whisker plots of physicochemical properties of FDA-Approved drugs (blue), FDA-Approved HIV protease inhibitors (purple), and new chemical compounds generated from COCONUT (orange), ChemDiv (red), and Enamine (green) fragment libraries, before applying physicochemical properties filtering. Table S2: Summary of the descriptive statistics of SlogP. Table S3: Summary of the descriptive statistics of MW. Table S4: Summary of the descriptive statistics of RB. Table S5: Summary of the descriptive statistics of TPSA. Table S6: Summary of the descriptive statistics of HBA. Table S7: Summary of the descriptive statistics of HBD. Table S8: Summary of the descriptive statistics of SAscore. Table S9: Summary of the descriptive statistics of solubility (Silicos-IT LowSw). Table S10: Summary of the descriptive statistics of lipophilicity (Consensus Log P). Table S11: Summary of the descriptive statistics of HIA. Table S12: Summary of the descriptive statistics of BBB permeability. Table S13: Percentage of compounds that are P-glycoprotein substrate, P-glycoprotein I inhibitor, and P-glycoprotein II inhibitor. Table S14: Percentage of compounds that inhibit the main cytochromes, CYP1A2, CYP2C19, CYP2C9, CYP2D6, CYP3A4. Table S15: Summary of the descriptive statistics of total clearance. Table S16: Summary of the descriptive statistics of toxicity descriptors.

Author Contributions: Designed and supervised the project, J.L.M.F; wrote the manuscript A.L.C.-H., J.L.M.-F.; methodology development, J.L.M.-F. and A.L.C.-H.; data curation and fragments generation, A.L.C.-H.; De novo design A.L.C.-H. and F.I.S.-G.; formal analysis A.L.C.-H. and J.L.M.-F; data visualization A. L. C.-H. and K.E.J.-M.; A.L.C.-H. and J.L.M.-F. All authors have read and agreed to the published version of the manuscript. 
Funding: Authors thank the Dirección General de Cómputo y de Tecnologías de Información y Comunicación (DGTIC), UNAM, for the computational resources to use Miztli supercomputer at UNAM under project LANCAD-UNAM-DGTIC-335.

Data Availability Statement: All data sets used in this study are available on https://figshare.com/s/ceb58d58e8f5585ce67e. TMAP_chemical_space_visualization.html; All_fragments_COCONUT_V4_184769.csv; HIV-protease_inhibitors_from_ChemDiv.csv; HIV_protease_inhibitors_from_COCONUT.csv; HIV_protease_inhibitors_from_Enamine.csv; FDA_APPROVED_DRUGS.csv; HIV_PROTEASE_INHIBITORS.csv; ADMETOX profiling_pkCSM.csv, ADMETOX profiling_swissadme.csv. Code used for generated new chemical compounds from chemical fragments are available on https://github.com/DIFACQUIM/De-novo-desing-of-HIV-1-inhibitors.

Acknowledgments: A.L.-C.H. and F.I.S.-G. thankful to CONACyT for the granted scholarship numbers 847870 and 848061, respectively. K.E.J.-M. thanks Dirección General de Asuntos del Personal Académico for the scholarship associated with the project PAPIIT IN201321. We thank Reymond's research group for developing Faerun library for Python.

Conflicts of Interest: The authors declare no conflict of interest.

\section{References}

1. HIV/AIDS Available online: https://www.who.int/news-room/fact-sheets/detail/hiv-aids. (accessed on Jul 15, 2021).

2. Zulfiqar, H.F.; Javed, A.; Sumbal; Afroze, B.; Ali, Q.; Akbar, K.; Nadeem, T.; Rana, M.A.; Nazar, Z.A.; Nasir, I.A.; et al. HIV Diagnosis and Treatment through Advanced Technologies. Front. Public Heal. 2017, 5, 32, doi:10.3389/fpubh.2017.00032.

3. Lv, Z.; Chu, Y.; Wang, Y. HIV protease inhibitors: a review of molecular selectivity and toxicity. HIV. AIDS. (Auckl). 2015, 7, 95104, doi:10.2147/HIV.S79956.

4. FDA Available online: https://www.fda.gov/consumers/free-publications-women/hiv-and-aids-medicines-help-you (accessed on Apr 28, 2021).

5. Schneider, G.; Clark, D.E. Automated de novo drug design: Are we nearly there yet? Angew. Chemie Int. Ed. 2019, 58, 1079210803, doi:10.1002/anie.201814681.

6. Torjesen, I. Drug Development: The journeyof a medicine from Lab to Shelf Available online: https://pharmaceutical-journal.com/article/feature/drug-development-the-journey-of-a-medicine-from-lab-to-shelf (accessed on May 29, 2021).

7. Medina-Franco, J.L. Grand challenges of computer-aided drug design: The road ahead. Front. Drug Discov. $2021,1,728551$. doi: $10.3389 /$ fddsv.2021.728551

8. Bung, N.; Krishnan, S.R.; Bulusu, G.; Roy, A. De novo design of new chemical entities for SARS-CoV-2 using artificial intelligence. Future Med. Chem. 2021, 13, 575-585, doi:10.4155/fmc-2020-0262.

9. Liu, X.; IJzerman, A.P.; van Westen, G.J.P. Computational approaches for de novo drug design: Past, present, and future. In Artificial Neural Networks; Cartwright, H., Ed.; Springer US: New York, NY, 2021; pp. 139-165 ISBN 978-1-0716-0826-5.

10. Mouchlis, V.D.; Afantitis, A.; Serra, A.; Fratello, M.; Papadiamantis, A.G.; Aidinis, V.; Lynch, I.; Greco, D.; Melagraki, G. Advances in De Novo Drug Design: From Conventional to Machine Learning Methods. Int. J. Mol. Sci. 2021, 22, 1676, doi:10.3390/ijms22041676.

11. Meyers, J.; Fabian, B.; Brown, N. De novo molecular design and generative models. Drug Discov. Today 2021, doi:10.1016/j.drudis.2021.05.019.

12. Devi, R.V.; Sathya, S.S.; Coumar, M.S. Evolutionary algorithms for de novo drug design - A survey. Appl. Soft Comput. 2015, 27, 543-552, doi:10.1016/j.asoc.2014.09.042.

13. Hartenfeller, M.; Schneider, G. De Novo Drug Design. In Chemoinformatics and Computational Chemical Biology; Bajorath, J., Ed.; Humana Press: Totowa, NJ, 2011; pp. 299-323 ISBN 978-1-60761-839-3.

14. Erlanson, D.A.; Fesik, S.W.; Hubbard, R.E.; Jahnke, W.; Jhoti, H. Twenty years on: the impact of fragments on drug discovery. Nat. Rev. Drug Discov. 2016, 15, 605-619, doi:10.1038/nrd.2016.109.

15. Osborne, J.; Panova, S.; Rapti, M.; Urushima, T.; Jhoti, H. Fragments: where are we now? Biochem. Soc. Trans. 2020, 48, 271-280, doi:10.1042/BST20190694.

16. Shinde, P.B.; Bhowmick, S.; Alfantoukh, E.; Patil, P.C.; Wabaidur, S.M.; Chikhale, R. V; Islam, M.A. De novo design based identification of potential HIV-1 integrase inhibitors: A pharmacoinformatics study. Comput. Biol. Chem. 2020, 88, 107319, doi:10.1016/j.compbiolchem.2020.107319.

17. Ghiandoni, G.M.; Bodkin, M.J.; Chen, B.; Hristozov, D.; Wallace, J.E.A.; Webster, J.; Gillet, V.J. Enhancing reaction-based de novo design using a multi-label reaction class recommender. J. Comput. Aided. Mol. Des. 2020, 34, 783-803, doi:10.1007/s10822-020-00300-6.

18. Saldívar-González, F.I.; Huerta-García, C.S.; Medina-Franco, J.L. Chemoinformatics-based enumeration of chemical libraries: a tutorial. J. Cheminform. 2020, 12, 64, doi:10.1186/s13321-020-00466-z.

19. Prado-Romero, D.L.; Medina-Franco, J.L. Advances in the exploration of the epigenetic televant chemical space. ACS Omega 2021, 6, 22478-22486, doi:10.1021/acsomega.1c03389. 
20. Atanasov, A.G.; Zotchev, S.B.; Dirsch, V.M.; et. al. Natural products in drug discovery: advances and opportunities. Nat. Rev. Drug Discov. 2021, 20, 200-216, doi:10.1038/s41573-020-00114-z.

21. Barnes, E.C.; Kumar, R.; Davis, R.A. The use of isolated natural products as scaffolds for the generation of chemically diverse screening libraries for drug discovery. Nat. Prod. Rep. 2016, 33, 372-381, doi:10.1039/c5np00121h.

22. Chávez-Hernández, A.L.; Sánchez-Cruz, N.; Medina-Franco, J.L. A Fragment Library of Natural Products and its Comparative Chemoinformatic Characterization. Mol. Inform. 2020, 39, 2000050, doi:10.1002/minf.202000050.

23. Karageorgis, G.; Foley, D.J.; Laraia, L.; Waldmann, H. Principle and design of pseudo-natural products. Nat. Chem. 2020, 12, 227-235, doi:10.1038/s41557-019-0411-x.

24. ChemDiv Available online: https://store.chemdiv.com/ (accessed on Jul 19, 2021).

25. Enamine Available online: https://enamine.net/compound-collections/fragment-collection (accessed on Jul 16, 2021).

26. Sorokina, M.; Steinbeck, C. Review on natural products databases: where to find data in 2020. J. Cheminform. 2020, 12, 20, doi:10.1186/s13321-020-00424-9.

27. Weininger, D. SMILES, a chemical language and information system. 1. Introduction to methodology and encoding rules. J. Chem. Inf. Comput. Sci. 1988, 28, 31-36, doi:10.1021/ci00057a005.

28. toolkit RDKit. Available online: http://rdkit.org (accessed on May 21, 2021).

29. MolVS. Available online: https://molvs.readthedocs.io/en/latest/ (accessed on May 21, 2021).

30. Lipinski, C.A.; Lombardo, F.; Dominy, B.W.; Feeney, P.J. Experimental and computational approaches to estimate solubility and permeability in drug discovery and development settings1PII of original article: S0169-409X(96)00423-1. The article was originally published in Advanced Drug Delivery Reviews 23 (1997) 3. Adv. Drug Deliv. Rev. 2001, 46, 3-26, doi:10.1016/S0169-409X(00)00129-0.

31. Veber, D.F.; Johnson, S.R.; Cheng, H.-Y.; Smith, B.R.; Ward, K.W.; Kopple, K.D. Molecular properties that influence the oral bioavailability of drug candidates. J. Med. Chem. 2002, 45, 2615-2623, doi:10.1021/jm020017n.

32. Lewell, X.Q.; Judd, D.B.; Watson, S.P.; Hann, M.M. RECAPRetrosynthetic combinatorial analysis procedure: a powerful new technique for identifying privileged molecular fragments with useful applications in combinatorial chemistry. J. Chem. Inf. Comput. Sci. 1998, 38, 511-522, doi:10.1021/ci970429i.

33. Zhao, Y.; Chen, C.-H.; Morris-Natschke, S.L.; Lee, K.-H. Design, synthesis, and structure activity relationship analysis of new betulinic acid derivatives as potent HIV inhibitors. Eur. J. Med. Chem. 2021, 215, 113287, doi:10.1016/j.ejmech.2021.113287.

34. Martin, D.E.; Salzwedel, K.; Allaway, G.P. Bevirimat: A novel maturation inhibitor for the treatment of hiv-1 infection. Antivir. Chem. Chemother. 2008, 19, 107-113, doi:10.1177/095632020801900301.

35. Lazerwith, S.E.; Siegel, D.; McFadden, R.M.; Mish, M.R.; Tse, W.C. 5.19 - New antiretrovirals for hiv and antivirals for HBV. In; Chackalamannil, S., Rotella, D., Ward, S.E.B.T.-C.M.C.I.I.I., Eds.; Elsevier: Oxford, 2017; pp. 628-664 ISBN 978-0-12-803201-5.

36. Qian, K.; Kuo, R.-Y.; Chen, C.-H.; Huang, L.; Morris-Natschke, S.L.; Lee, K.-H. Anti-AIDS Agents 81. Design, synthesis, and structure-activity relationship study of betulinic acid and moronic acid derivatives as potent HIV maturation inhibitors. J. Med. Chem. 2010, 53, 3133-3141, doi:10.1021/jm901782m.

37. Huang, Q.; Chen, H.; Luo, X.; Zhang, Y.; Yao, X.; Zheng, X. Structure and anti-HIV activity of betulinic acid analogues. Curr. Med. Sci. 2018, 38, 387-397, doi:10.1007/s11596-018-1891-4.

38. Rogers, D.; Hahn, M. Extended-connectivity fingerprints. J. Chem. Inf. Model. 2010, 50, 742-754, doi:10.1021/ci100050t.

39. Durant, J.L.; Leland, B.A.; Henry, D.R.; Nourse, J.G. Reoptimization of MDL Keys for use in drug discovery. J. Chem. Inf. Comput. Sci. 2002, 42, 1273-1280, doi:10.1021/ci010132r.

40. Probst, D.; Reymond, J.-L. Visualization of very large high-dimensional data sets as minimum spanning trees. J. Cheminform. 2020, 12, 12, doi:10.1186/s13321-020-0416-x.

41. TMAP Available online: https://tmap.gdb.tools/ (accessed on Sep 14, 2021).

42. Greener, J.G.; Kandathil, S.M.; Moffat, L.; Jones, D.T. A guide to machine learning for biologists. Nat. Rev. Mol. Cell Biol. 2021, doi:10.1038/s41580-021-00407-0.

43. Sánchez-Cruz, N.; Pilón-Jiménez, B.A.; Medina-Franco, J.L. Functional group and diversity analysis of BIOFACQUIM: A Mexican natural product database. F1000Research 2020, 8, doi:10.12688/f1000research.21540.2.

44. Chávez-Hernández, A.L.; Sánchez-Cruz, N.; Medina-Franco, J.L. Fragment library of natural products and compound databases for drug discovery. Biomolecules 2020, 10, 1518, doi:10.3390/biom10111518.

45. Ertl, P.; Schuffenhauer, A. Estimation of synthetic accessibility score of drug-like molecules based on molecular complexity and fragment contributions. J. Cheminform. 2009, 1, 8, doi:10.1186/1758-2946-1-8.

46. Wishart, D.S.; Feunang, Y.D.; Guo, A.C.; Lo, E.J.; Marcu, A.; Grant, J.R.; Sajed, T.; Johnson, D.; Li, C.; Sayeeda, Z.; et al. DrugBank 5.0: a major update to the DrugBank database for 2018. Nucleic Acids Res. 2018, 46, D1074-D1082, doi:10.1093/nar/gkx1037.

47. Daina, A.; Michielin, O.; Zoete, V. SwissADME: A free web tool to evaluate pharmacokinetics, drug-likeness and medicinal chemistry friendliness of small molecules. Sci. Rep. 2017, 7, 1-13, doi:10.1038/srep42717.

48. Pires, D.E.V.; Blundell, T.L.; Ascher, D.B. pkCSM: Predicting small-molecule pharmacokinetic and toxicity properties using graph-based signatures. J. Med. Chem. 2015, 58, 4066-4072, doi:10.1021/acs.jmedchem.5b00104.

49. Durán-Iturbide, N.A.; Díaz-Eufracio, B.I.; Medina-Franco, J.L. In silico ADME/Tox profiling of natural products: A focus on BIOFACQUIM. ACS Omega 2020, 5, 16076-16084, doi:10.1021/acsomega.0c01581.

50. Saldívar-González, F.I.; Lenci, E.; Calugi, L.; Medina-Franco, J.L.; Trabocchi, A. Computational-aided design of a library of lactams through a diversity-oriented synthesis strategy. Bioorg. Med. Chem. 2020, 28, 115539, doi:10.1016/j.bmc.2020.115539. 
51. Laurini, R. 5 - Geographic Relations. In; Laurini, R.B.T.-G.K.I., Ed.; Elsevier, 2017; pp. 83-109 ISBN 978-1-78548-243-4.

52. Ganesan, A. The impact of natural products upon modern drug discovery. Curr. Opin. Chem. Biol. 2008, 12, 306-317, doi:10.1016/j.cbpa.2008.03.016.

53. Tinworth, C.P.; Young, R.J. Facts, Patterns, and principles in drug discovery: Appraising the Rule of 5 with measured physicochemical data. J. Med. Chem. 2020, 63, 10091-10108, doi:10.1021/acs.jmedchem.9b01596.

54. Bergström, C.A.S.; Yazdanian, M. Lipophilicity in drug development: Too much or not enough? AAPS J. 2016, 18, 1095-1100, doi:10.1208/s12248-016-9947-5.

55. Smith, D.A.; Beaumont, K.; Maurer, T.S.; Di, L. Clearance in drug design. J. Med. Chem. 2019, 62, 2245-2255, doi:10.1021/acs.jmedchem.8b01263. 